\title{
Blind test comparison of the performance and wake flow between two in-line wind turbines exposed to different turbulent inflow conditions
}

\author{
Jan Bartl and Lars Sætran \\ Department of Energy and Process Engineering, Norwegian University of Science and Technology, \\ Trondheim, 7491, Norway \\ Correspondence to: Jan Bartl (jan.bartl@ntnu.no)
}

Received: 23 August 2016 - Discussion started: 5 September 2016

Revised: 3 December 2016 - Accepted: 16 January 2017 - Published: 9 February 2017

\begin{abstract}
This is a summary of the results of the fourth blind test workshop that was held in Trondheim in October 2015. Herein, computational predictions on the performance of two in-line model wind turbines as well as the mean and turbulent wake flow are compared to experimental data measured at the wind tunnel of the Norwegian University of Science and Technology (NTNU). A detailed description of the model geometry, the wind tunnel boundary conditions and the test case specifications was published before the workshop. Expert groups within computational fluid dynamics (CFD) were invited to submit predictions on wind turbine performance and wake flow without knowing the experimental results at the outset. The focus of this blind test comparison is to examine the model turbines' performance and wake development with nine rotor diameters downstream at three different turbulent inflow conditions. Aside from a spatially uniform inflow field of very low-turbulence intensity $(\mathrm{TI}=0.23 \%)$ and high-turbulence intensity $(\mathrm{TI}=10.0 \%)$, the turbines are exposed to a grid-generated highly turbulent shear flow $(\mathrm{TI}=10.1 \%)$.

Five different research groups contributed their predictions using a variety of simulation models, ranging from fully resolved Reynolds-averaged Navier-Stokes (RANS) models to large eddy simulations (LESs). For the three inlet conditions, the power and the thrust force of the upstream turbine is predicted fairly well by most models, while the predictions of the downstream turbine's performance show a significantly higher scatter. Comparing the mean velocity profiles in the wake, most models approximate the mean velocity deficit level sufficiently well. However, larger variations between the models for higher downstream positions are observed. Prediction of the turbulence kinetic energy in the wake is observed to be very challenging. Both the LES model and the IDDES (improved delayed detached eddy simulation) model, however, consistently manage to provide fairly accurate predictions of the wake turbulence.
\end{abstract}

Given the constraints of transmission and installation costs, the available area for offshore wind farm installations is fairly limited. Under these circumstances wake interactions play an important role when evaluating the energy production since the energy captured by an upstream wind turbine leaves significantly less energy in the wake for the downstream turbine. For certain wind directions these power losses are estimated to be up to $10-20 \%$ for large offshore wind farms
(Barthelmie et al., 2009). Furthermore, the rotor-generated turbulence in the wake is a source of augmented material fatigue on the downstream rotor.

In order to be able to come up with holistic control approaches for optimizing a wind farm, well-performing prediction tools for the wake flow behind a wind turbine rotor for all kinds of atmospheric conditions are needed. Therefore, the development of simple wake models already began in the early 1980s. Analytical wake models by Jensen (1983), Ainslie (1988), Crespo et al. (1988), Frandsen et al. (2006) 
and Larsen et al. (2008) are based on a number of simplifications and are calibrated with empirical parameters. Most of the state-of-the-art software used for industrial wind farm planning is still based on these engineering wake models. However, they are not able to reconstruct the wake characteristics in a sufficient degree of detail (Sanderse et al., 2011).

With an increase in computational power, advanced computational fluid dynamics (CFD) models based on more fundamental physics arose. These CFD models are computationally more expensive but are able to resolve the flow structures in much larger detail. In general, two types of CFD approaches are state of the art in wake modeling: Reynoldsaveraged Navier-Stokes (RANS) equations that average the turbulent fluctuations and the computationally more expensive large eddy simulations (LESs), which solve for large eddies only. Hybrid models like detached eddy simulations (DESs) combine the advantages of calculating unsteady flow effects from LES as well as resolving small scales in the boundary layers like RANS does. Another challenge is the modeling of the interaction of the wind turbine rotor with the flow: the rotor geometry can either be fully resolved or simplified as a two-dimensional force field. The latter option is usually more efficient with respect to computational time. In RANS models it is possible to fully resolve the rotor geometry and thus model complex three-dimensional flow. In LES models, however, a full resolution of the rotor geometry is difficult because the smaller scales that determine the forces at the interaction surface are not resolved. Thus, the rotor is often modeled as a two-dimensional force field, which requires detailed knowledge of the lift and drag forces that act under certain inflow conditions.

Even though the wake behind full-scale wind turbines was recently measured (Kocer et al., 2011; Kumer et al., 2015; Trujillo et al., 2016), the unsteady inflow conditions in fullscale experiments make it very difficult to use those data to verify wake prediction models. Therefore, wind tunnel experiments on model turbines under controlled boundary conditions are an appropriate method for verifying simulation tools.

Despite the drawbacks of low Reynolds numbers and possible wall blockage effects in model experiments, a number of well-defined comparison tests have been conducted. One of the first model-scale experiments was the investigation by Talmon (1985). The wake was measured on a small rotor with a diameter of $D=0.36 \mathrm{~m}$ in order to serve as a reference experiment for calculations. In addition to uniform inflow, the wake development was studied in a simulated atmospheric boundary layer. Another seminal investigation was conducted by Medici and Alfredsson (2006). With three-dimensional wake flow measurements on a $D=0.18 \mathrm{~m}$ model turbine down to $x / D=9$, they shed light on phenomena like wake rotation, wake deflection in yawed operation and bluff body vortex shedding frequencies from the rotor.

At the Norwegian University of Science and Technology (NTNU) two model turbines of the rotor diameter
$D=0.90 \mathrm{~m}$ were extensively investigated. Adaramola and Krogstad (2011) analyzed the effect of modifying tip speed ratio, blade pitch angle and yaw angle on a downstream turbine. Eriksen (2016) investigated the three-dimensional rotor-generated turbulence in the wake of one model turbine in detail. Bartl et al. (2012) examined the wake behind two model turbines, while special attention to asymmetries and wake rotation was given by Schümann et al. (2013). A recent study by Bartl and Sætran (2016) investigated the interrelation of wake flow and the performance of a downstream turbine for axial-induction-based wind farm control methods.

The largest rotor investigated for wake comparison studies was the MEXICO rotor, with a diameter of $4.5 \mathrm{~m}$ (Schepers et al., 2010), in which the rotor performance as well as the wake flow were examined in detail. A second campaign investigating even more effects, including span-wise pressure distributions, yaw misalignment and unsteady effects, was realized at a large German-Dutch Wind Tunnel (DNW). A benchmark comparison of the comprehensive set of measurement data with numerical calculations is found in Schepers et al. (2014).

In 2011 the first blind test workshop on turbine performance and wake development behind one model turbine was organized. The geometry of the model turbine and wind tunnel environment was made available to the public, and dedicated research groups were invited to predict the model turbine's performance and the wake development up to $x / D=5.0$ rotor diameters downstream. A total of 11 sets of predictions were submitted and reported by Krogstad and Eriksen (2013). This first blind test experiment showed a significant scatter in the performance predictions, with a variation of several magnitudes in predictions of turbulent quantities in the wake between the different contributions. Therefore, it was decided to perform another blind test workshop in 2012, increasing the test complexity by adding a second turbine aligned with the upstream turbine. The participants were asked to predict the performance of both turbines as well as the wake behind the downstream turbine. Nine different submissions were received, showing clear variations in the quality of the predictions between the different modeling methods (Pierella et al., 2014). For a third blind test workshop held in 2013, the complexity was increased slightly again. The two model wind turbines were positioned with a spanwise offset of half a rotor diameter. The results reported by Krogstad et al. (2014) showed that a LES simulation method proved to simulate this complex flow case fairly well. For the present fourth blind test workshop held in Trondheim in October 2015, the focus was directed on the effect of different turbulent inflow conditions on the performance of an aligned two-turbine setup. Test cases of low turbulent uniform inflow, highly turbulent inflow as well as nonuniform highly turbulent shear are investigated. The wake flow behind the upstream turbine is analyzed, which defines the inflow conditions to the downstream turbine. Five different groups contributed CFD simulations ranging from RANS to 


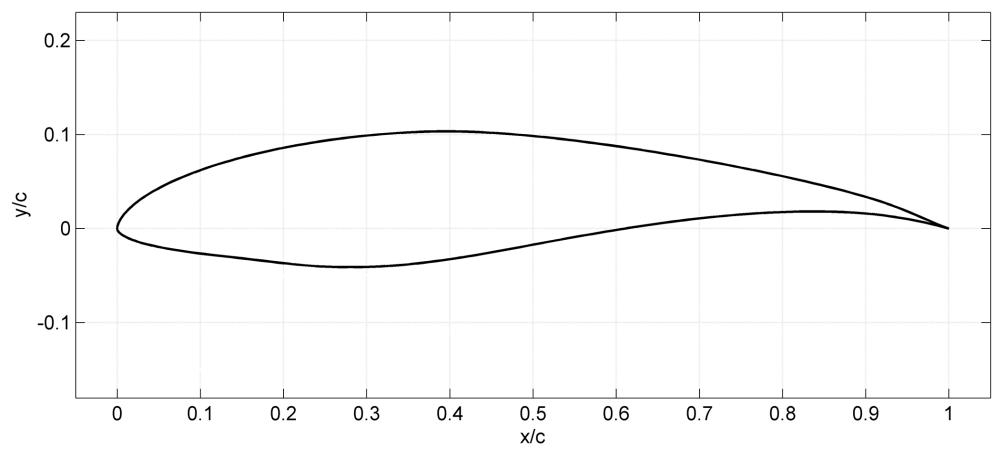

Figure 1. NREL S826 airfoil geometry.

LES to DES computations. Although a general improvement in the results is observed over the years, this report shows the strengths and drawbacks of the different modeling methods and underlines the persistent importance of validation of CFD codes with well-defined experimental datasets.

\section{Methods}

\subsection{Test case description}

\subsubsection{Wind tunnel}

The experimental data of this study are measured in the closed-loop wind tunnel at NTNU in Trondheim. The rectangular test section of the wind tunnel is $2.71 \mathrm{~m}$ broad, $1.81 \mathrm{~m}$ high and $11.15 \mathrm{~m}$ long. The wind tunnel roof is adjusted for a zero pressure gradient, generating a constant velocity in the entire test section. The wind tunnel inlet speed is controlled by an inlet contraction, which is equipped with static pressure holes at the circumferences at two defined cross sections. The wind tunnel is driven by a $220 \mathrm{~kW}$ fan located downstream of the test section, able to generate maximum wind speeds of up to $U_{\max }=30 \mathrm{~m} \mathrm{~s}^{-1}$.

\subsubsection{Model turbines, rotor and airfoil characteristics}

The model wind turbines have a three-bladed rotor with diameters of $D_{\mathrm{T} 1}=0.944 \mathrm{~m}$ and $D_{\mathrm{T} 2}=0.894 \mathrm{~m}$. The small difference in rotor diameter stems from a slightly different hub geometry of the rigs. Apart from that the blade geometry is exactly the same. Both turbines rotate counter-clockwise when observed from an upstream point of view. The rotors are both driven by a $0.37 \mathrm{~kW}$ AC Siemens electric motor and controlled by a Siemens Micromaster 440 frequency inverter. The motor rotational speed can be varied from about 100 to $3000 \mathrm{rpm}$, while the generated power is burned off by an external load resistance.

The turbine blades were designed using the NREL S826 airfoil from the root to the tip. The airfoil, as shown in Fig. 1, was designed at the National Renewable Energy Laboratory (NREL) and a detailed description of the airfoil's characteristics is given by Somers (2005). Herein, the geome- try is specified and the performance characteristics are estimated. Lift and drag coefficients are presented for a range of operating Reynolds numbers $\left(\operatorname{Re}_{\mathrm{C}, \mathrm{tip}, F S}=10^{6}\right)$ for a fullscale turbine, which are 1 order of magnitude higher than the Reynolds numbers prevailing in this model experiment $\left(R e_{\mathrm{C}, \text { tip, model }}=10^{5}\right)$. In order to be able to also characterize the airfoil's performance at model-scale Reynolds numbers, a number of two-dimensional experiments on airfoil performance have been conducted. Sarmast and Mikkelsen (2013) performed an experiment on a two-dimensional S826 wing section of the chord length $c_{\mathrm{L}}=0.10 \mathrm{~m}$ at DTU in Denmark. They observed hysteretic behavior for $\operatorname{Re}_{\mathrm{C}}<1 \times 10^{5}$, which is assumed to be the cause for Reynolds-dependent behavior of the inner blade elements of the upstream turbine under design conditions. Another experimental set of S826 airfoil data was presented by Ostovan et al. (2013) from Middle East Technical University (METU) in Turkey. They investigated lift and drag coefficients from $R e_{\mathrm{C}}=7.15 \times 10^{4}$ to $R e_{\mathrm{C}}=1.45 \times 10^{5}$ on a two-dimensional wing with a chord length of $c_{\mathrm{L}}=0.20 \mathrm{~m}$. No hysteretic effects for low Reynolds numbers were found in this experiment. A third experimental set of airfoil characteristics from $R e_{\mathrm{C}}=7.00 \times 10^{4}$ to $R e_{\mathrm{C}}=6.00 \times 10^{5}$ was measured by Aksnes (2015) on a wing section of $c_{\mathrm{L}}=0.45 \mathrm{~m}$ at NTNU, Norway. No Reynoldsdependent behavior was found at low Reynolds numbers in this experiment either. The measured lift and drag coefficients of these three experiments are in good agreement in the linear lift region, while in the pre-stall and stall regions, significant differences between the three datasets are present. For $R e_{\mathrm{C}}=10^{5}$ DTU's measurements predict stall already at $\alpha \approx 8^{\circ}$, while in METU's and NTNU's experiments, stall presents later around $\alpha \approx 11^{\circ}$. Furthermore, somewhat higher lift values are measured in NTNU's dataset in the prestall region compared to the other datasets. Numerical simulations by Sagmo et al. (2016) as well as Prytz et al. (2017) point out strong three-dimensional flow effects caused by stall cells in the pre-stall and stall regions. This could be a possible cause for varying experimental results in this region.

Both rotors are designed for an optimum tip speed ratio of $\lambda_{\mathrm{T} 1}=\lambda_{\mathrm{T} 2}=6.0$. The blades are milled from aluminium and 


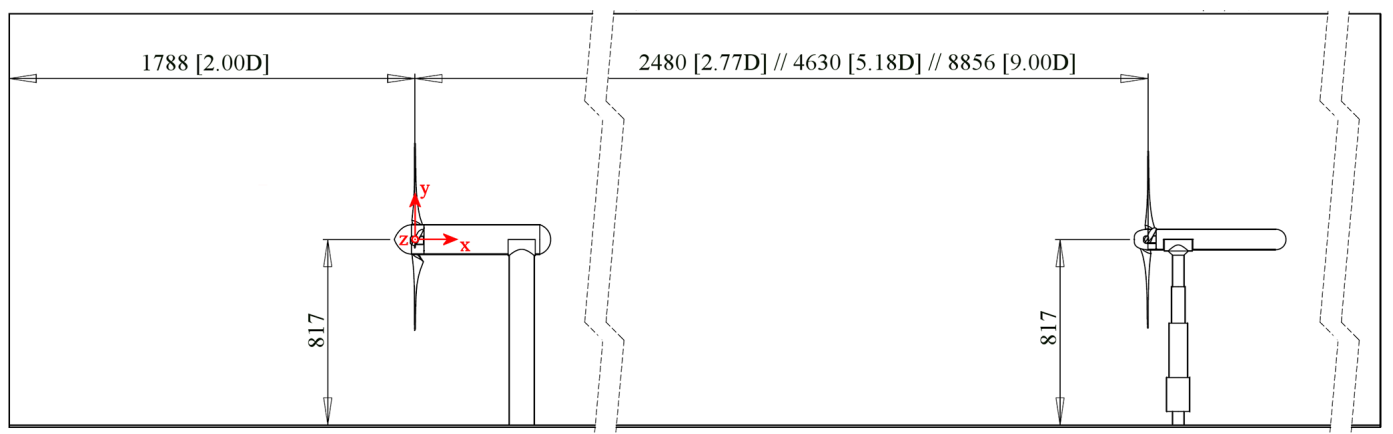

Figure 2. Setup of the model wind turbines in the wind tunnel and reference coordinate system.

(a)

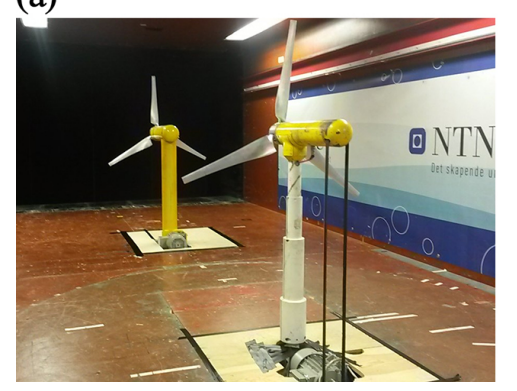

(b)

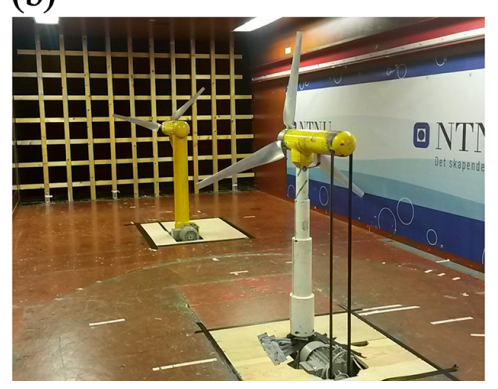

(c)

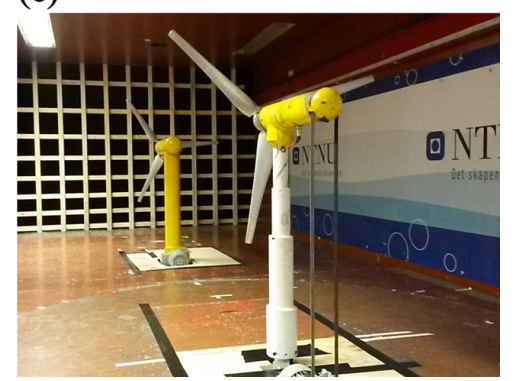

Figure 3. Test case A: low-turbulence uniform inflow (a); test case B: high-turbulence uniform inflow (b); test case C: high-turbulence shear inflow (c).

the blade tips are cut straight. More details about the blade geometry, such as detailed chord and twist data, are found in an invitational document by Sætran and Bartl (2015).

In this blind test experiment the model turbines are positioned at the wind tunnel center line. The upstream turbine T1's rotor plane is located at $x / D=2.00$ from the test section inlet, which is verified to be far enough away to not affect the reference velocity measurement at the inlet contraction. The downstream turbine $\mathrm{T} 2$ is positioned at 2.77 and $5.18 \mathrm{D}$, $9.00 \mathrm{D}$ downstream of the upstream turbine rotor. The hub height of both turbines is adjusted to $h_{\text {hub }}=0.817 \mathrm{~m}$. In Fig. 2 a side cut of the wind tunnel is shown, indicating a reference coordinate system and the wind turbine positions.

\subsubsection{Inflow conditions}

For this blind test experiment three different turbulent inflow conditions are investigated. This is supposed to shed light on the effects of various turbulence levels, as well as shear in the atmosphere, on the performance of a wind turbine and its wake. As it is almost impossible to create realistic conditions that resemble atmospheric stability classes in a wind tunnel environment, simplified cases of turbulent inflow are created.

The first inflow condition investigated is a uniform inflow of very low turbulence and is described from here on as test case A. As shown in Fig. 3a, there is no grid installed at the inlet of the test section, resulting in a clean and uniform flow.
Hot-wire measurements at the upstream turbine position give a turbulence intensity level of $\mathrm{TI}=0.23 \%$ on an integral turbulent length scale of $L_{u u}=0.045 \mathrm{~m}$. Over the rotor swept area, the mean velocity in the empty tunnel is found to be uniform to within $\pm 0.6 \%$. The boundary layer thickness at wind tunnel walls was measured to be $y_{\mathrm{BL}}=0.200 \mathrm{~m}$ at the upstream turbine position.

In order to investigate the effects of turbulence on wind turbine performance and wake development, the measurements of test case B are performed using a large-scale turbulence grid at the inlet to the test section (Fig. 3b). The biplanar grid has a solidity of $35 \%$ and is built from wooden bars with a $47 \mathrm{~mm} \times 47 \mathrm{~mm}$ cross section. The grid mesh size is $M=0.240 \mathrm{~m}$, which generates a turbulence intensity of $\mathrm{TI}=10.0 \%$ at the position of the upstream turbine. The integral length scale here is assessed from an autocorrelation of a hot-wire time series and is calculated to be $L_{u u}=0.065 \mathrm{~m}$ at this position. The grid produces considerable span-wise variations in the flow, but as soon as the flow reaches the position of the upstream turbine T1, the mean velocity is measured to be uniform to within $\pm 1.5 \%$ over the rotor area. The turbulence intensity is also assessed to be constant to within $\pm 1.0 \%$. In this grid-generated turbulent flow, the turbulent kinetic energy decays with increasing distance from the grid. As the flow reaches the first position of the downstream turbine $\mathrm{T} 2,2.77 \mathrm{D}$ downstream of $\mathrm{T} 1$, the turbulence intensity 
in the empty tunnel decays to $\mathrm{TI}=4.8 \%$, while the integral length scale increases to $L_{u u}=0.100 \mathrm{~m}$.

In a third test case $\mathrm{C}$, the effect of shear flow combined with high turbulence is investigated. For this purpose a largescale shear-flow-generating turbulence grid is installed at the inlet of the test section, as shown in Fig. 3c. The horizontal mesh width is constant at $M_{\mathrm{h}}=0.240 \mathrm{~m}$, while the vertical mesh heights vary between $M_{\mathrm{v}, \min }=0.016 \mathrm{~m}$ near the floor and $M_{\mathrm{v}, \max }=0.300 \mathrm{~m}$ underneath the roof. The grid is biplanar and has a solidity of $38 \%$. As for the evenly spaced turbulence grid, it is again built from wooden bars with a $47 \mathrm{~mm} \times 47 \mathrm{~mm}$ cross section. At the position of the upstream turbine T1, a turbulence intensity of $10.1 \%$ is measured at hub height. The turbulent length scale is estimated to be $L_{u u}=0.097 \mathrm{~m}$ for this case. The kinetic energy in the flow decays with the distance from the grid. The turbulence intensity decays to $\mathrm{TI}=5.2 \% 2.77 \mathrm{D}$ further downstream, while the length scale increases to $L_{u u}=0.167 \mathrm{~m}$. At $5.18 \mathrm{D}$ downstream of $\mathrm{T} 1$, the turbulence intensity decays to $\mathrm{TI}=4.1 \%$, while at $9.00 \mathrm{D}$ only $\mathrm{TI}=3.7 \%$ remains.

Because wind shear and turbulence are generated only at the grid position at the tunnel inlet, their development throughout the tunnel is measured for all turbine positions. Wind shear can be described by the power law in Eq. (1), which expresses the wind speed $U$ as a function of height $y$, provided that the wind speed at an arbitrary reference height $y_{\text {ref }}$ is known:

$$
\frac{U}{U_{\mathrm{ref}}}=\left(\frac{y}{y_{\mathrm{ref}}}\right)^{\propto}
$$

The power law coefficient $\alpha$ describes the strength of shear in the wind profile. A wind profile based on a shear coefficient of about $\alpha=0.11$ is chosen for this experiment, resembling the shear at typical stable atmospheric conditions (Hsu et al., 1994), although the grid-generated turbulence in the wind tunnel is much higher than in a stable boundary layer. The mean and turbulent flow profiles at all relevant positions are shown in Fig. 4.

During the present experiments, the reference wind speed was kept constant at $U_{\text {ref }}=11.5 \mathrm{~m} \mathrm{~s}^{-1}$, which is tested to give a Reynolds-number-independent turbine performance for all inflow conditions. Since the downstream turbine T2 experiences significantly lower average wind speeds when operating in the turbulent wake, Reynolds-numberindependent performance characteristics are measured down to an inflow velocity of $U_{\text {inflow }}=6.0 \mathrm{~m} \mathrm{~s}^{-1}$ at $\mathrm{TI}=5.0 \%$ background turbulence.

For test case $\mathrm{C}$, in which the velocity increases with height, the reference velocity of $U_{\text {ref }}=11.5 \mathrm{~m} \mathrm{~s}^{-1}$ is set at the turbine hub height $h_{\text {hub }}=0.817 \mathrm{~m}$. This reference height is chosen for simplicity reasons, although the rotor-equivalent wind speed (Wagner et al., 2014) that represents the center of kinetic power in the shear inflow is found to be slightly below the turbine hub height (Maal, 2014).

\subsection{Experimental methods}

\subsubsection{Power and thrust measurements}

Both model turbines are equipped with a HBM torque transducer of the type T20W-N/2-Nm, which is connected to the rotor shaft through flexible couplings. In addition, an optical photo cell is installed on the shaft, giving a defined peak signal for every full rotation of the rotor. After subtracting the measured friction in the ball bearing between the rotor and torque sensor, the mechanical power in the rotor shaft can be calculated. The power in both turbines is measured and controlled simultaneously to ensure a stable operation of both turbines.

The thrust force is measured by a six-component force balance produced by Carl Schenck AG. The drag force on the tower and nacelle structure is first measured without the rotor being present. Thus, it is possible to assess the rotor thrust by subtracting the tower-nacelle drag from the total drag.

\subsubsection{Wake flow measurements}

The mean and turbulent velocities in the wake behind the upstream turbine $\mathrm{T} 1$ are measured by a single hot-wire anemometer (HWA) in constant temperature mode (CTA). Each measurement point is sampled for $45 \mathrm{~s}$ at $20 \mathrm{kHz}$, resulting in a total of $9.0 \times 10^{5}$ samples. The signals are amplified and filtered appropriately to avoid distortion by noise, for example. All the wake measurements are repeated using a twocomponent laser Doppler anemometry (LDA) system from Dantec Dynamics for verification. A time series of $5.0 \times 10^{4}$ samples is sampled for a varying period of about $30 \mathrm{~s}$. The reference velocity $U_{\text {ref }}$ used for normalization of the mean and turbulent wake velocity, as well as the nondimensional power and thrust coefficients, is measured at the inlet contraction of the wind tunnel. The pressure difference around the circumferences of two defined cross sections is logged simultaneously for every measuring point. The air density $\rho$ in the experiment is calculated from the measured air temperature and atmospheric pressure in the test section for every measurement point.

\subsubsection{Statistical measurement uncertainties}

The statistical uncertainty of every sample of the power, thrust and mean velocity measurements is calculated following the procedure proposed by Wheeler and Ganji (2004). Random errors are computed from the standard deviations of the various measured signals on a $95 \%$ confidence interval. Also taking systematic errors from the calibration procedures into account by following the procedure of Eriksen (2016), a total error is calculated. Herein, the systematic error of about $\pm 1.0 \%$ from the velocity calibration is seen to be the major contributor to the total uncertainty. The uncertainty in the turbulent quantities in the wake flow is calculated according to the approach of Benedict and Gould (1996). 
(a)

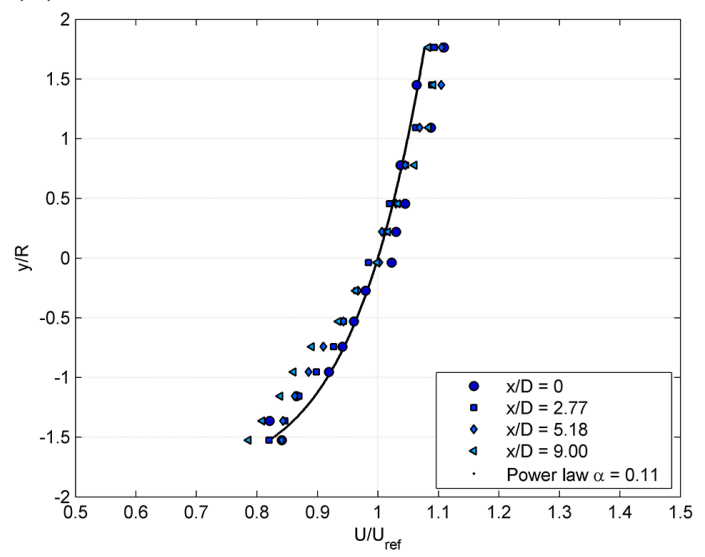

(b)

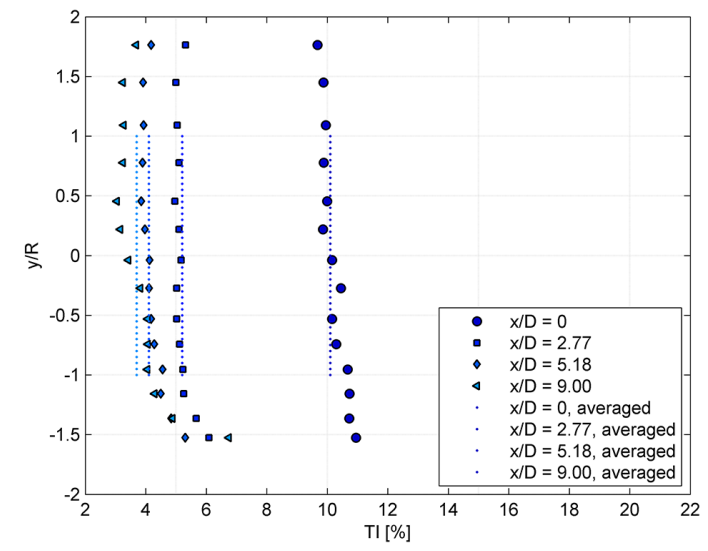

Figure 4. Measured and rotor-averaged values of normalized mean velocity $U / U_{\text {ref }}$ (a) and turbulence intensity TI [\%] (b) at the position of $\mathrm{T} 1(x / D=0)$ and the positions of $\mathrm{T} 2(x / D=0,2.77,5.18,9.00)$ in the empty tunnel for test case $\mathrm{C}$ (shear flow grid).

Table 1. Overview of simulation methods and parameters. Abbreviations for rotor models: actuator line (ACL), blade element momentum (BEM), fully resolved rotor (FRR). Abbreviations for flow models: improved delayed detached eddy simulation (IDDES), large eddy simulation (LES), Reynolds-averaged Navier-Stokes simulation (RANS).

\begin{tabular}{|c|c|c|c|c|c|c|c|c|}
\hline & & $\begin{array}{l}\text { Simulation } \\
\text { software }\end{array}$ & $\begin{array}{l}\text { Rotor } \\
\text { model }\end{array}$ & $\begin{array}{l}\text { Airfoil } \\
\text { data }\end{array}$ & $\begin{array}{l}\text { Flow or turbulence } \\
\text { model }\end{array}$ & $\begin{array}{l}\text { Mesh } \\
\text { properties }\end{array}$ & $\begin{array}{l}\text { Number of } \\
\text { cells or nodes }\end{array}$ & $\begin{array}{l}\text { Tunnel } \\
\text { blockage }\end{array}$ \\
\hline UU-DTU & $D$ & EllipSys3D & ACL & Exp. DTU & LES & Cartesian & $2.9 \times 10^{7}$ cells & Yes \\
\hline Vrije (flow) & $\square$ & OpenFOAM & FRR & - & RANS $k-\omega$ & Hexahedral & $3.5 \times 10^{7}$ cells & Yes \\
\hline Vrije (forces) & $\square$ & Matlab & BEM & XFoil & - & - & - & - \\
\hline LUT (ACL) & & ANSYS CFX & ACL & XFoil & RANS $k-\omega$ SST & Arbitrary & $3.7 \times 10^{6}$ nodes & Yes \\
\hline LUT (FRR) & & ANSYS CFX & FRR & - & RANS $k-\omega$ SST & Structured & $4.2 \times 10^{4}$ nodes & Yes \\
\hline CD-adapco & 负 & Star-CCM+ & FRR & - & IDDES Sp.-Al. & Hexah./Polyh. & $2.5 \times 10^{7}$ cells & Yes \\
\hline CMR & $<$ & Music & BEM & XFoil & RANS $k-\varepsilon$ & Structured & $5.0 \times 10^{5}$ nodes & Yes \\
\hline
\end{tabular}

The uncertainty in the upstream turbine power coefficient at design conditions is calculated to be within $\pm 3.0 \%$, while it is lower than $\pm 2.0 \%$ for the thrust coefficient. It is observed that the uncertainty of the mean velocity is somewhat larger in the free stream outside the wake. At higher velocities the sensitivity of the hot-wire probe is smaller, which produces higher uncertainties. The measured values of the turbulent kinetic energy are observed to feature the highest uncertainty in the shear layer between wake and free stream flow.

\subsection{Computational methods}

The computational methods applied by the five different contributors are described in the following subsections. Furthermore, an overview of the different simulation methods and parameters is presented in Table 1.

\subsubsection{Uppsala University and DTU (UU-DTU)}

S. Sarmast, R. Mikkelsen and S. Ivanell from Uppsala University, Campus Gotland, Sweden, and Technical University of Denmark (DTU), Campus Lyngby, Denmark, contributed with a dataset simulated by LES methods combined with an ACL approach. The DTU in-house code EllipSys3D, which is based on a multi-block finite volume approach, was used to solve the Navier-Stokes computations. The convective terms are herein discretized by a combination of third-order and a fourth-order schemes. The resolution of the time domain is defined small enough, that a blade tip moves less than a half cell size per time step. The flow field around the wind turbine rotor was simulated using the actuator line technique developed by Sørensen and Shen (2002). Herein, the Navier-Stokes equations are solved with body forces distributed along rotating lines representing the blades of the wind turbine. The lift and drag coefficients are taken from the previously mentioned self-generated dataset for the NREL S826 airfoil by Sarmast and Mikkelsen (2013). For each of 
the 43 blade points the forces are interpolated for the local Reynolds numbers in a range of 40000 to 120000 . Additionally, a force line is introduced to account for the drag force generated by the tower. The wake flow field is calculated by solving the Navier-Stokes equations using LES with an integrated sub-grid-scale (SGS) viscosity model.

A regular Cartesian grid, which is divided into 875 blocks, makes out the computational domain. With 32 points in each block and 43 points representing each blade, a total of 28.6 million mesh points are used to simulate the various test cases. This resolution was tested to give a grid-independent simulation result.

The inlet turbulence is modeled by implanting synthetically resolved turbulent fluctuations $1.5 \mathrm{D}$ upstream of the position of the upstream rotor $\mathrm{T} 1$. These fluctuations from a pre-generated turbulence field are superimposed to the mean velocities through momentum sources, yielding isotropic homogenous turbulence. The mean and turbulent profiles of the different test cases are tested to give a good match with the corresponding wind tunnel values. In addition, the effect of shear flow combined with high turbulence is investigated. The shear profile is implemented to match the profile given in the invitational document by Sætran and Bartl (2015). A more detailed description of the method can be found in Sarmast et al. (2014).

\subsubsection{Vrije University Brussels (Vrije)}

N. Stergiannis from Vrije University and Von Karman Institute (VKI) in Brussels, Belgium, performed Reynoldsaveraged Navier-Stokes (RANS) simulations using the opensource software package OpenFOAM in combination with a multiple rotating frame (MRF) approach. Therein, the full rotor geometry is resolved in its own frame of reference and the flow is calculated around the "frozen rotor". The subdomain is connected to the stationary frame of reference by an arbitrary mesh interface (AMI). A grid independency test was executed investigating different cell sizes, giving an independent result with a total number of $3.5 \times 10^{7}$ cells. Slip conditions are used at the wind tunnel walls, which was deemed to save computational effort and still takes into account the blockage effect generated by the walls. The rotor and the nacelle are completely resolved, but the turbine towers are not simulated in the final computations. The boundary layers on the blades and nacelle are resolved down to $y^{+} \approx 30$. The standard $k-\omega$ turbulence model as implemented in OpenFOAM v.2.4 is applied for the presented simulations. The mean and turbulent inlet velocities were matched with the experimental values provided in the invitational document. Because the blade forces could not be directly extracted from the fully resolved rotor simulations, a blade element momentum (BEM) code based on the method by Ning (2014) was used to calculate the power and thrust characteristics of the model wind turbines. The lift and drag coefficients are computed with the open-source software XFoil (Drela, 2013) for the NREL S826 airfoil at all prevailing Reynolds numbers. The reference velocity for the downstream turbine is calculated as the average velocity over a line of one radius $x / D=1$ upstream of the downstream rotor. Only test cases $\mathrm{A}$ and $\mathrm{B}$ are modeled.

\subsection{3 Łódź University of Technology (LUT)}

M. Lipian, M. Karczewski and P. Wiklak from the Institute of Turbomachinery at Łódź University of Technology, Poland, contributed two datasets computed by the commercial CFD software ANSYS CFX. All simulations were performed to find a steady state solution of the RANS equations using the $k-\omega$ SST model for turbulence closure.

For test cases A, B and C they fully resolved the rotor geometry. Thus, the solver resolves the actual flow around the rotor and no additional assumptions needed to be made. These simulations will be denoted as fully resolved rotor model LUT (FRR) from now on. Two rotating subdomains are established around the rotors, while the main wind tunnel domain is stationary. A structural mesh is created with the software ICEM CFD to discretize the domains. The wind tunnel is discretized by a total number of $3.0 \times 10^{4}$ plus two refined subdomains around the rotors of $6.0 \times 10^{3}$ nodes each. A grid independence test was executed for the rotor subdomain to prove grid-independent convergence.

For the test cases $\mathrm{B}_{1}, \mathrm{~B}_{2}$ and $\mathrm{B}_{3}$ a different approach was chosen. The rotors are represented by a custom-made actuator line model, which will be denoted as LUT (ACL). Herein, the blades are modeled as parallel epipedons, representing a subdomain in which the RANS equations are modified. The flow is modified by an addition of force components, which are calculated from tabulated lift and drag data dependent on the local chord and angle of attack. The lift and drag data are taken from the invitational document and were originally created with XFoil. Furthermore, the ACL model includes a Prandtl tip-loss correction. For these test cases an unstructured mesh is used in the wind tunnel main domain and parallel epipedon around the blades, discretized by a total number of $1.7 \times 10^{6}$ nodes in the main domain plus 2 times $1.0 \times 10^{6}$ nodes in the subdomains around the rotors. Because the test cases $\mathrm{B}$ and $\mathrm{B}_{2}$ are identical, a direct comparison between the performance and wake results of the FRR and ACL simulations is possible.

\subsubsection{CD-adapco (CD-adapco)}

S. Evans and J. Ryan from CD-adapco, London, United Kingdom, contributed a full dataset of predictions simulated by improved delayed detached eddy simulations (IDDES). The IDDES Spalart-Allmaras turbulence model is used for turbulence closure in the boundary layers. Both the meshing and the actual simulation are carried out with their commercial software package STAR-CCM+, which is a finitevolume solver using cells of arbitrary shape. 
Table 2. Overview of turbine operating conditions downstream turbine positions as well as wake measurement positions for the five different test cases.

\begin{tabular}{llrrrrc}
\hline $\begin{array}{l}\text { Test } \\
\text { case }\end{array}$ & Inflow & $\begin{array}{r}\text { Inlet turbulence } \\
\text { at position of T1 }\end{array}$ & $\begin{array}{r}\text { Tip speed } \\
\text { ratio } \lambda_{\mathrm{T} 1}\end{array}$ & $\begin{array}{r}\text { Position } x / D \text { of } \\
\text { downstream turbine } \mathrm{T} 2\end{array}$ & $\begin{array}{r}\text { Tip speed } \\
\text { ratio } \lambda_{\mathrm{T} 2}\end{array}$ & $\begin{array}{c}\text { Wake measurement } \\
\text { position at } x / D\end{array}$ \\
\hline $\mathrm{A}$ & uniform & $0.23 \%$ & 6.0 & 5.18 & 4.5 & 2.77 \\
$\mathrm{~B} 1$ & uniform & $10.0 \%$ & 6.0 & 2.77 & 4.5 & - \\
$\mathrm{B} 2$ & uniform & $10.0 \%$ & 6.0 & 5.18 & 4.5 & - \\
$\mathrm{B} 3$ & uniform & $10.0 \%$ & 6.0 & 9.00 & 4.5 & $2.77 / 5.18 / 8.50$ \\
$\mathrm{C}$ & shear & $10.1 \%$ & 6.0 & 5.18 & 4.5 & 2.77 \\
\hline
\end{tabular}

Aside from the turbine rotors, the exact geometry of the turbine nacelles, towers and wind tunnel walls is modeled. The computational domain is divided into three subdomains. In the main wind tunnel domain, a hexahedral dominant grid is applied, which is further refined around the turbines and in the wake region. In the disc-shaped regions around the rotors, an isotropic polyhedral mesh of even finer resolution is utilized. The boundary layers around the blade surfaces are resolved down to $y^{+}<2$. The rotating disk domains around the turbine rotors are connected to the main domain via an arbitrary sliding interface. For the entire computational domain, around $2.5 \times 10^{7}$ grid cells are applied.

The inlet conditions are modeled with the synthetic eddy method, generating an inflow field of defined turbulence intensity and length scales that correspond to the values given in the invitational document. For test case $\mathrm{C}$, a shear flow is defined by a power law at the wind tunnel inlet. Explicit transient modeling is used to simulate the wind turbine interactions, while the turbine rotations are modeled as a rigid body motion. A transient second-order model with a time step of $\mathrm{d} t=1.0 \times 10^{-4} \mathrm{~s}$ is used. Advanced limiter options for minimum limiting and higher-order spatial schemes are used in a segregated solver. The transient calculation is run for $1 \mathrm{~s}$ in test cases $A, B_{1}, B_{2}$ and $C$ and $2.5 \mathrm{~s}$ in test case $B_{3}$ due to the higher separation distance. The required values are thereafter averaged for a time period of $0.5 \mathrm{~s}$.

More information about the use of Star-CMM+ in rotating flows can be found in Mendonça et al. (2012), for example.

\subsubsection{CMR Instrumentation (CMR)}

A. Hallanger and I. Ø. Sand from CMR Instrumentation in Bergen, Norway, provided a dataset based on RANS simulations combined with a BEM approach. For the calculation of the mean and turbulent flow quantities, their in-house CFD code called Music was used. The RANS equations are solved with a standard $k-\varepsilon$ model with Launder-Spalding coefficients. Furthermore, a sub-grid turbulence model is applied to represent the rotor-generated turbulence. Therein, it is assumed that the production rate of turbulent kinetic energy and its rate of dissipation are integrated over the wake of the wind turbine and distributed over the near field. Convective and diffusive fluxes are approximated with the second- order Van Leer (1974) and central difference schemes. The turbulent intensity and length scales at the inlet are specified according to the experimental values given in the invitational document for the three different test cases. For test case C, a power law profile is used.

The rotors are included as sub-models in the CFD code. They are represented by their reaction forces on the flow field. The blade forces are simulated by a BEM code, including wake rotation. The blades are divided into 30 blade elements in radial direction. The BEM code includes the Prandtl tip-loss correction as well as Glauert's empirical model for highly loaded rotors. The lift and drag coefficients were calculated from the software XFoil (Drela, 2013) depending on angle of attack, Reynolds number and relative turbulence intensity. Therein, the transition amplification numbers $\left(N_{\text {crit }}\right)$ represent the turbulence intensity levels present at the different positions in the wind tunnel. Three-dimensional corrections for two-dimensional force coefficients according to the BEM method by Ning (2014) were applied. These forces were used as source terms for axial and rotational momentum conservation. The turbine hubs and towers were modeled as flow resistances in the same control volume as the rotors. Turbine hubs were represented by a drag coefficient of $C_{\mathrm{D}, \mathrm{hub}}=0.6$, while the tower drag was approximated by $C_{\text {D,tower }}=1.2$.

Wind tunnel walls were modeled by wall functions. The entire wind tunnel environment including the two rotors was resolved in a total of $5 \times 10^{5}$ structured grid nodes. Steady state simulations of the blade forces were performed with an angular increment of $15^{\circ}$, resulting in a total of 24 azimuthal positions of the turbine rotors. This was deemed to be sufficient to include the effects of shear flow on the first turbine. A detailed description of the computational methods applied is given in Hallanger and Sand (2013).

\subsection{Required output}

In total, five different test cases are provided for simulation in this blind test experiment. An overview of the turbine operating conditions and position as well as the measurement station of the wake measurements is shown in Table 2. 


\subsubsection{Wind turbine performance}

For all five test cases the power coefficients $C_{\mathrm{P}, \mathrm{T} 1}$ and $C_{\mathrm{P}, \mathrm{T} 2}$ (Eq. 2) as well as the thrust coefficients $C_{\mathrm{T}, \mathrm{T} 1}$ and $C_{\mathrm{T}, \mathrm{T} 2}$ (Eq. 3) of both turbines are compared:

$$
\begin{aligned}
C_{\mathrm{P}, \mathrm{T} 1 / \mathrm{T} 2} & =\frac{8 P_{\mathrm{T} 1 / \mathrm{T} 2}}{\rho \pi D_{\mathrm{T} 1 / \mathrm{T} 2}^{2} U_{\mathrm{ref}}^{3}}, \\
C_{\mathrm{T}, \mathrm{T} 1 / \mathrm{T} 2} & =\frac{8 F_{\mathrm{T} 1 / \mathrm{T} 2}}{\rho \pi D_{\mathrm{T} 1 / \mathrm{T} 2}^{2} U_{\mathrm{ref}}^{2}} .
\end{aligned}
$$

Herein, $P_{\mathrm{T} 1 / \mathrm{T} 2}$ denotes the mechanical power of the turbine shaft, $F_{\mathrm{T} 1 / \mathrm{T} 2}$ the thrust force in the stream-wise direction on the rotor and $\rho$ the air density. The upstream turbine $\mathrm{T} 1$ is operated at a tip speed ratio of $\lambda_{\mathrm{T} 1}=$ $\omega \times D_{\mathrm{T} 1} / 2 \times U_{\text {ref }}=6.0$, whereas the downstream turbine T2 is run at $\lambda_{\mathrm{T} 2}=\omega \times D_{\mathrm{T} 2} / 2 \times U_{\text {ref }}=4.5$. Note that the same reference velocity $U_{\text {ref }}$ defined at the test section inlet is used for both turbines. The optimal tip speed ratio for the downstream turbine $\mathrm{T} 2$ is also $\lambda_{\mathrm{T} 2}=\lambda_{\mathrm{T} 1}=6.0$ when the turbine is unobstructed. Since T2 operates in the wake, the velocity that was actually experienced was considerably lower, also reducing the optimal rotational speed and thus the tip speed ratio $\lambda_{\mathrm{T} 2}$. The optimal tip speed ratio at which the maximum power $P_{\mathrm{T} 2}$ is achieved in fact varies between $\lambda_{\mathrm{T} 2}=4.0$ and 5.0 depending on the turbine separation distance $x / D$ and inlet turbulence level $\mathrm{TI}_{\text {Inlet }}$. For better comparability, a fixed tip speed ratio of $\lambda_{\mathrm{T} 2}=4.5$ was chosen.

\subsubsection{Mean and turbulent wake flow}

Furthermore, the horizontal profiles of the mean and turbulent flows are compared at the predefined wake measurement positions (Table 2). The upstream turbine is still operated at $\lambda_{\mathrm{T} 1}=6.0$ for all five test cases. The profiles of the normalized mean velocity $U^{*}$ (Eq. 4) and the normalized turbulent kinetic energy $k^{*}$ (Eq. 5) are calculated at the turbine hub height $h_{\text {hub }}=0.817 \mathrm{~m}$ :

$U^{*}=U / U_{\mathrm{ref}}$,

$k^{*}=k / U_{\text {ref }}^{2}$.

In a Cartesian coordinate system the turbulent kinetic energy $k$ is defined as

$k=\frac{1}{2}\left(u_{x}^{\prime 2}+u_{y}^{\prime 2}+u_{z}^{\prime 2}\right)$.

According to Bruun (1995), the HWA measures an effective cooling velocity $U_{\text {eff }}$ that can be described by the Jørgensen equation (Eq. 7).

$U_{\mathrm{eff}}^{2}=U_{x}^{2}+k U_{y}^{2}+h U_{z}^{2}$

Depending on the magnitude of the flow velocity, the coefficients $k$ and $h$ typically have values around 1.05 and
0.2 (Bruun, 1995), which means that $U_{\text {eff }}$ can be approximated by the velocity perpendicular to the wire. For flows with $U_{x} \gg U_{y}$, the effective cooling velocity has the same magnitude as the stream-wise component $U_{x}$, which is in this case a reasonable assumption for wake measurements at downstream positions starting at $x / D=2.77$.

Therefore, the isotropic normal stress approximation (Eq. 8) is used to determine the turbulent kinetic energy in each measurement point:

$k=\frac{3}{2} u_{x}^{\prime 2}$

This approximation is most certainly not appropriate for the zones with high anisotropy, but Krogstad et al. (2014) showed that the isotropic normal stress approximation is a well-fitting approximation in the turbine wake. They measured all three components of the stress tensor with a crosswire probe for one wake profile at $x / D=1$. Furthermore, they demonstrated a very good agreement of the isotropic approximation and the component-wise calculation of $k$.

For the LDA measurements the stream-wise and crosswise flow components $U_{x}$ and $U_{z}$ are measured. Since the stress tensors $u_{x}^{\prime}$ and $u_{z}^{\prime}$ from these measurements are seen to be very isotropic, the turbulent kinetic energy $k$ is also in this case approximated by the stream-wise stress $u_{x}^{\prime}$ only (Eq. 8).

The computed values of mean velocity as well as turbulent kinetic energy from HWA and LDA measurements compare very well. In regions of increased rotation, as in the wake center, the HWA consistently predicts slightly lower mean velocity values. Here, the influence of binormal cooling velocity $U_{y}$ is more pronounced, though not really significant.

\subsection{Comparative methods}

\subsubsection{Direct comparison of turbine performances}

The predictions of the power coefficients $C_{\mathrm{P}, \mathrm{T} 1}$ and $C_{\mathrm{P}, \mathrm{T} 2}$ as well as the thrust coefficients $C_{\mathrm{T}, \mathrm{T} 1}$ and $C_{\mathrm{T}, \mathrm{T} 2}$ at the predefined operating points are directly compared to the experimentally measured values in graphs and tables. The deviations from the measured reference value are discussed on a percentage basis in the text.

\subsubsection{Statistical performance measures for wake prediction}

The predictions of the mean and turbulent wake flow $U^{*}$ and $k^{*}$ are compared in graphs to the measured profiles from the HWA and LDA experiments. In order to provide a more general comparison of the predictions with the experimental results, statistical performance measures are computed as proposed by Chang and Hanna (2004). These measures include the fractional bias (FB), the normalized mean square error (NMSE), the geometric mean bias (MG), the geometric variance $(\mathrm{VG})$ and the correlation coefficient $(R)$. For this 
purpose, the predictions are compared to the experimental measurements using HWA in the exact same locations as the 41 measurement points along a horizontal line at a hub height from $z / R=-2.0$ to $z / R=2.0$. Thus, the following statistical performance measures are calculated and compared in tables for each test case:

$$
\begin{aligned}
& \mathrm{FB}=\frac{\overline{x_{\mathrm{m}}}-\overline{x_{\mathrm{p}}}}{0.5\left(\overline{x_{\mathrm{m}}}+\overline{x_{\mathrm{p}}}\right)}, \\
& \mathrm{NMSE}=\frac{\overline{\left(x_{\mathrm{m}}-x_{\mathrm{p}}\right)^{2}}}{\overline{x_{\mathrm{m}}} \times \overline{x_{\mathrm{p}}}}, \\
& \mathrm{MG}=\exp \left(\overline{\ln x_{\mathrm{m}}}-\overline{\ln x_{\mathrm{p}}}\right), \\
& \mathrm{VG}=\exp \left[\overline{\left(\ln x_{\mathrm{m}}-\ln x_{\mathrm{p}}\right)^{2}}\right], \\
& R=\frac{\overline{\left(x_{\mathrm{m}}-\overline{x_{\mathrm{m}}}\right) \times\left(x_{\mathrm{p}}-\overline{x_{\mathrm{p}}}\right)}}{\sigma_{x_{\mathrm{m}}} \times \sigma_{x_{\mathrm{p}}}} .
\end{aligned}
$$

Herein, $x_{\mathrm{m}}$ are the measured values and $x_{\mathrm{p}}$ the values predicted by the models. In this case the compared values $x$ are the normalized mean velocity $U^{*}=u / U_{\text {ref }}$ and normalized turbulent kinetic energy $k^{*}=k / U_{\text {ref }}^{2}$. The overbar $\bar{x}$ means that an average over all the data points from $z / R=-2$ to $z / R=2$ is taken, and $\sigma_{x}$ refers to the standard deviation of the dataset from $z / R=-2$ to $z / R=2$.

A perfect model prediction would result in a FB and NMSE of zero and MG, VG and $R$ of 1 . It has to be stated that these statistical performance measures can by no means give a comprehensive evaluation of the performance of a model, but only provide a general correlation of all data points.

FB and MG are measures of the systematic error, while FB is measured on a linear scale and MG is based on a logarithmic scale. Note that it still might be possible to get a perfect correlation using FB and MG even though the single points are far off at the specific measurement locations. Conversely, NMSE and VG represent the scatter in the correlation of measured and predicted data and include both systematic and random errors (Chang and Hanna, 2004). Finally, the widely used correlation coefficient $R$ indicates the linear correlation between the measured and predicted values. In this study it is the only measure that directly compares the predicted and measured values at a specific location. Since $R$ is insensitive to addition or multiplication of constants, it is often not recommended as a stand-alone value for the evaluation of a model (Chang and Hanna, 2004). For the comparison in this blind test experiment, however, the correlation coefficient $R$ is deemed a robust method. The addition or multiplication of the predicted values is in most cases not relevant in the prevailing test cases. All predictions start from the same predefined boundary conditions, meaning that there is not a big offset in most data.

\section{Results}

The comparisons of the predictions and experimental results are analyzed for the different inflow conditions. In Sect. 3.1, power, thrust and wake predictions for test case A (lowturbulence inflow) are presented. Thereafter, all the test cases for high-turbulence inflow conditions for all three separation distances (test cases $\mathrm{B}_{1}, \mathrm{~B}_{2}$. $\mathrm{B}_{3}$ ) are analyzed in Sect. 3.2. Finally, the results of test case $\mathrm{C}$, featuring a highly turbulent shear flow, are compared in Sect. 3.3.

Experimental results for power and thrust are indicated by filled black circles for the upstream turbine and empty circles for the downstream turbine. The measurements of the wake profiles using HWA are marked with filled black circles, while flow measurements using LDA are indicated by filled grey circles. The different contributions of numerical simulations are assigned one consistent symbol and color for power, thrust and wake flow predictions.

\subsection{Test case A: low-turbulence uniform inflow}

\subsubsection{Power and thrust predictions}

The power and thrust predictions for test case A (lowturbulence inflow, $\mathrm{TI}=0.23 \%$ ) from the five contributions are compared to the experimental results in Fig. 5. The respective numerical values are listed in Table 3.

The experimentally measured power coefficient of the upstream turbine has its maximum $C_{\mathrm{P}, \max }=0.462$ at $\lambda=6.0$ and its runaway tip speed ratio at $\lambda=11.1$. At a turbine tip speed ratio of about $\lambda=3.5$, a rapid transition of $C_{\mathrm{P}, \mathrm{T} 1}$ into stall is observed.

The predictions of the power coefficient of the upstream turbine $\mathrm{T} 1$ at its design operating point $\lambda_{\mathrm{T} 1}=6.0$ show a scatter of about $\pm 7 \%$ compared to the measured $C_{\mathrm{P}, \mathrm{T} 1}$. This points out significant differences in the modeling methods. While CMR generated a Reynolds-dependent dataset for liftand drag coefficients using the airfoil design and analysis code XFoil (Drela, 2013) as an input for their BEM model, UU-DTU used an experimentally generated lift and drag dataset produced by Sarmast and Mikkelsen (2013) as an input for their ACL model. Another aspect is how the predictions modeled the influence of solid wall blockage on the $C_{\mathrm{P}}$ values. Because the flow cannot expand freely around the turbine, the induction is reduced, resulting in higher power production of the turbine than that in an unblocked flow. All five contributions took the wind tunnel boundaries into account, resulting in fairly good approximations of the upstream turbine's $C_{\mathrm{P}}$ at design conditions.

The scatter in $C_{\mathrm{P}}$ for the downstream turbine T2 is considerably larger than for T1. T2 is operated around its design point at $\lambda_{\mathrm{T} 2}=4.5$ (referring to $U_{\text {ref }}$ measured upstream of $\mathrm{T} 1$ ) in the wake at a separation distance of $x / D_{\mathrm{T} 2}=5.18$ from the upstream turbine $\mathrm{T} 1$. The power is underestimated by up to $25 \%$ and overpredicted by no more than $30 \%$. How- 
(a)

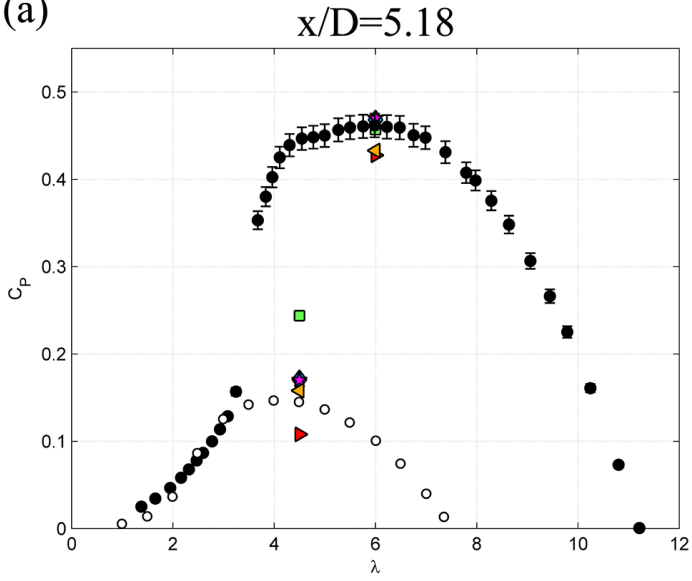

(b)

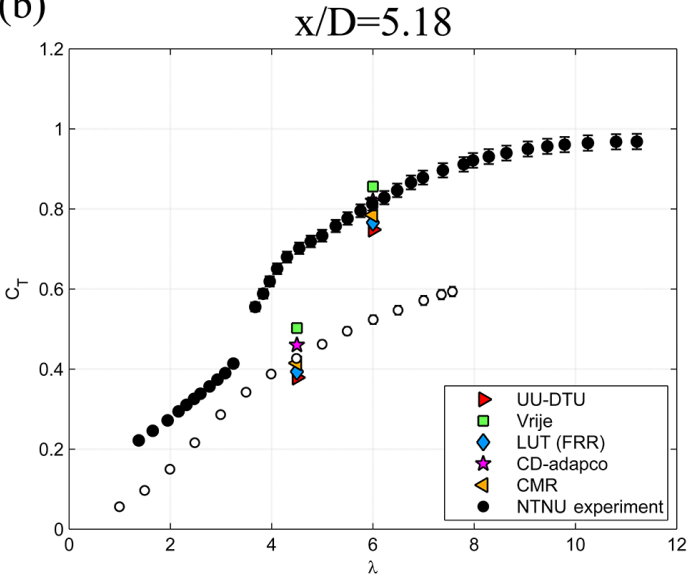

Figure 5. Power coefficient $C_{\mathrm{P}}$ (a) and thrust coefficient $C_{\mathrm{T}}$ (b) for T1 (filled circles) and T2 (empty circles) compared for test case A. The downstream turbine $\mathrm{T} 2$ is positioned at $x / D=5.18$ downstream of $\mathrm{T} 1$, and the upstream turbine $\mathrm{T} 1$ is operated at $\lambda_{\mathrm{T} 1}=6.0$. The reference velocity is $U_{\text {ref }}=11.5 \mathrm{~m} \mathrm{~s}^{-1}$.

Table 3. Numerical values of power coefficient $C_{\mathrm{P}}$ and thrust coefficient $C_{\mathrm{T}}$ for test case $\mathrm{A}$. The downstream turbine T2 is positioned at $5.18 D$ downstream of $\mathrm{T} 1$. $\mathrm{T} 1$ is operated at $\lambda_{\mathrm{T} 1}=6.0$ and $\mathrm{T} 2$ is operated at $\lambda_{\mathrm{T} 1}=4.5$, referring to the far upstream reference velocity $U_{\text {ref }}=11.5 \mathrm{~m} \mathrm{~s}^{-1}$.

\begin{tabular}{llllll}
\hline & & \multicolumn{2}{c}{ Upstream turbine T1 } & \multicolumn{2}{c}{ Downstream turbine T2 } \\
& & $C_{\mathrm{P}, \mathrm{T} 1}$ & $C_{\mathrm{T}, \mathrm{T} 1}$ & $C_{\mathrm{P}, \mathrm{T} 2}$ & $C_{\mathrm{T}, \mathrm{T} 2}$ \\
\hline UU-DTU & $\checkmark$ & 0.428 & 0.748 & 0.108 & 0.379 \\
Vrije & $\square$ & 0.457 & 0.856 & 0.244 & 0.502 \\
LUT (FRR) & $\searrow$ & 0.468 & 0.766 & 0.171 & 0.394 \\
CD-adapco & $\searrow$ & 0.470 & 0.820 & 0.170 & 0.460 \\
CMR & $\triangleleft$ & 0.433 & 0.785 & 0.158 & 0.415 \\
Experiment & $\bullet$ & 0.462 & 0.811 & 0.145 & 0.427 \\
\hline
\end{tabular}

ever, some predictions such as CMR, LUT and CD-adapco manage to match the experimental result reasonably well, overestimating the downstream turbine power by only 9$17 \%$. This is a rather small deviation given the large scatter of more than $100 \%$ observed in previous blind test experiments (Pierella et al., 2014; Krogstad et al., 2015).

The predictions of the thrust coefficient for turbines $\mathrm{T} 1$ and T2 give a similar picture, as shown in Fig. 5b. Even though the upstream turbine thrust is slightly underpredicted by most simulations, the scatter is significantly smaller than in earlier blind tests. The $C_{\mathrm{T}}$ predictions for the downstream turbine show approximately the same scatter as the upstream turbine. The BEM predictions by CMR matched the experimental results very closely for both turbines.

\subsubsection{Wake predictions}

For the low inlet turbulence test case A, predictions of the wake flow at $x / D_{\mathrm{T} 2}=2.77$ behind the upstream turbine are compared. Horizontal profiles of the normalized mean ve- locity $U^{*}$ and the normalized turbulent kinetic energy $k^{*}$ are compared at hub height, as shown in Fig. 6a and b.

As already observed in a very similar test case in blind test 1 (Krogstad and Eriksen, 2013), the mean velocity profile at $x / D=2.77$ features two distinct minima located behind the blade tips of the rotor (Fig. 6a). The evident asymmetry in the wake center is caused by the advection of the tower wake into the swirling rotor wake as shown in rotor wake experiments by Schümann et al. (2013). The wake shape and levels of velocity deficit are very well predicted by CDadapco and UU-DTU, reflected in well-matching statistical performance measures, as presented in the left part of Table 4. Aside from small error values of $\mathrm{FB}_{U^{*}}$ and $\mathrm{NMSE}_{U^{*}}$, the correlation coefficient scores of $R_{U^{*}, \mathrm{CD} \text {-adapco }}=0.960$ and $R_{U^{*}, \text { UU-DTU }}=0.927$ score significantly better than the other predictions. CD-adapco's IDDES simulations furthermore manage to capture the shape of the wake profile very well, including the asymmetries caused by the tower wake in the center of the profile. Another good prediction of the two minima and correct wake deficit levels is given by the fully resolved rotor simulations by LUT. However, the ver- 
(a)

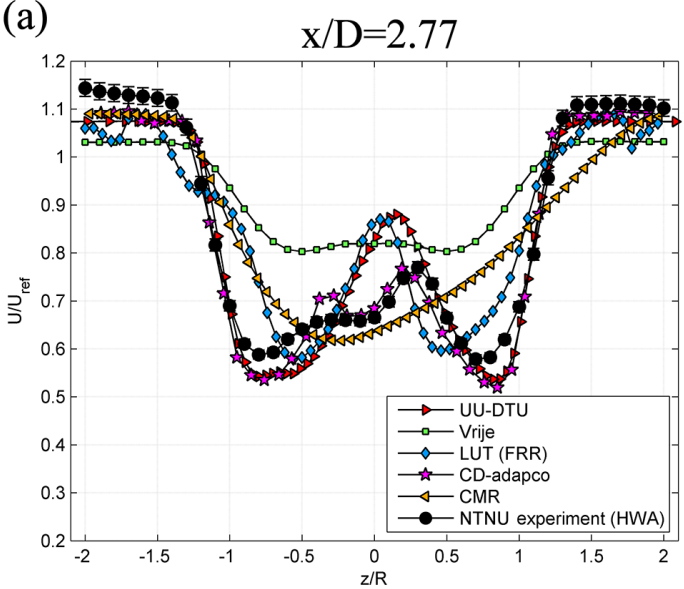

(b)

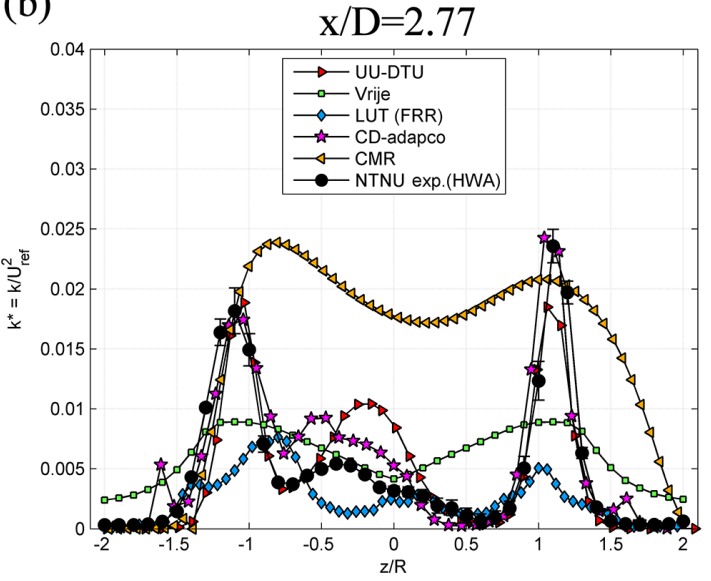

Figure 6. Normalized mean velocity $U / U_{\text {ref }}$ (a) and normalized turbulent kinetic energy $k / U_{\text {ref }}$ (b) in the wake $x / D=2.77$ behind T1 measured for test case A. The upstream turbine T1 is operated at $\lambda_{\mathrm{T} 1}=6.0$. The reference velocity is $U_{\text {ref }}=11.5 \mathrm{~m} \mathrm{~s}$.

Table 4. Statistical performance measures FB, NMSE, MG, VG and $R$ of the normalized mean velocity $U^{*}$ and normalized turbulent kinetic energy $k^{*}$ predictions of the five different models for test case A. The wake flow is predicted at stream-wise measurement position $x / D=2.77$ downstream of $\mathrm{T} 1$.

\begin{tabular}{lrrrrrrrrrrr}
\hline & & $\mathrm{FB}_{U^{*}}$ & $\mathrm{NMSE}_{U^{*}}$ & $\mathrm{MG}_{U^{*}}$ & $\mathrm{VG}_{U^{*}}$ & $R_{U^{*}}$ & $\mathrm{FB}_{\mathrm{k}^{*}}$ & $\mathrm{NMSE}_{\mathrm{k}^{*}}$ & $\mathrm{MG}_{\mathrm{k}^{*}}$ & $\mathrm{VG}_{\mathrm{k}^{*}}$ & $R_{\mathrm{k}^{*}}$ \\
\hline UU-DTU & $\square$ & 0.031 & 0.001 & 1.032 & 1.010 & 0.927 & -0.047 & 0.002 & 1.797 & 6.828 & 0.870 \\
Vrije & $\square$ & -0.081 & 0.007 & 0.897 & 1.041 & 0.895 & -0.218 & 0.048 & 0.411 & 6.038 & 0.669 \\
LUT (FRR) & -0.009 & 0.000 & 0.980 & 1.017 & 0.877 & 0.675 & 0.515 & 1.522 & 1.879 & 0.547 \\
CD-adapco & $\longleftarrow$ & 0.042 & 0.002 & 1.047 & 1.006 & 0.960 & -0.206 & 0.043 & 0.918 & 2.528 & 0.938 \\
CMR & $\$ & 0.000 & 0.000 & 0.988 & 1.016 & 0.886 & -1.019 & 1.404 & 0.338 & 89.922 & 0.378 \\
\hline
\end{tabular}

tical wake extension as modeled by LUT is too small for this low-turbulence inflow test case, reflected in a somewhat lower correlation coefficient of $R_{U^{*}, \mathrm{LUT}}=0.877$. CMR's RANS simulations based on a $k-\varepsilon$ turbulence model predict a Gaussian wake shape with only one minimum already at $x / D=2.77$ downstream of the rotor, suggesting a much more homogenous flow, as measured in the experiments. A slightly poorer correlation coefficient of $R_{U^{*} \text {,LUT }}=0.877$ is therefore calculated. Integrating over CMR's mean wake profile, however, gives a fair estimate of the kinetic energy contained in the wake flow, which is seen in error values $\mathrm{FB}_{U^{*}, \mathrm{CMR}}$ and $\mathrm{NMSE}_{U^{*}, \mathrm{CMR}}$ that are approximately zero, as well as $\mathrm{MG}_{U^{*}, \mathrm{CMR}}$ and $\mathrm{VG}_{U^{*}, \mathrm{CMR}}$, which are close to the perfect model value 1 . The reason for that is that these measures do not specifically take the measurement location into account, but are calculated based on different averages over the entire wake. Vrije's method does not resolve the details in the mean velocity profile because the turbine tower was not included in the simulation. The velocity deficit in the wake is significantly underestimated; in average it amounts to only about $50 \%$ of the experimentally measured values. Still, a fairly good correlation coefficient of $R_{U^{*} \text {,Vrije }}=0.895$ is computed. This unexpectedly high value might be due to the fact that the correlation coefficient is insensitive to addition and multiplication of constants, as discussed by Chang and Hanna (2004). This is confirmed by significantly higher deviations of Vrije's prediction in $\mathrm{FB}_{U^{*}}, \mathrm{NMSE}_{U^{*}}, \mathrm{MG}_{U^{*}}$ and $\mathrm{VG}_{U^{*}}$ from the perfect model than the other models.

The normalized turbulent kinetic energy profiles are compared in Fig. 6b. The experimental profile shows two distinct peaks in the shear layer generated by the tip vortices around $z / R= \pm 1$. A third, substantially smaller peak slightly left of the wake center is ascribed to the turbulence generated by the tower and nacelle structures. It can be observed that the turbulent kinetic energy in the shear layer is very well predicted by UU-DTU's LES as well as CD-adapco's IDDES model, which both match the turbulence peaks generated by the tip vortices perfectly. The statistical performance measures of the turbulence predictions of all models, as presented in the right part of Table 4, show a similar picture as previously observed in the mean velocity predictions. CD-adapco predicts the turbulence profile very well, resulting in a high correlation coefficient of $R_{\mathrm{k}^{*} \text {, CD-adapco }}=0.938$. The slightly lower correlation of UU-DTU's profile $\left(R_{\mathrm{k}^{*}, \mathrm{UU} \text {-DTU }}=0.870\right)$ is mainly due to an overprediction of the turbulence generated by the tower in the center of the wake. LUT's RANS sim- 
ulation based on the $k-\omega$ SST turbulence model shows the three distinct peaks but underpredicts the turbulence levels significantly. This is underlined by considerably higher error values of $\mathrm{FB}_{\mathrm{k}^{*} \text {,LUT }}=0.675$ and $\mathrm{NMSE}_{\mathrm{k}^{*} \text {,LUT }}=0.515$ than in the other simulations. Vrije's simulations based on a $k-\omega$ turbulence model indicate the two peaks in the shear layer; however, these predictions also give far too low of turbulent kinetic energy (TKE) values in the shear layer. In the unaffected free stream flow, however, Vrije's model predicts a significantly too-high TKE, although the free stream turbulence should be predefined as an input value. Therefore, a slightly poorer correlation coefficient of $R_{\mathrm{k}^{*} \text {, Vrije }}=0.669$ is calculated, while the geometrical variance of the turbulence profile with $\mathrm{VG}_{\mathrm{k}^{*} \text {, Vrije }}=6.038$ is rather high. CMR's simulation shows two TKE peaks in the shear layer of the same magnitude as in the experimental dataset. However, the turbulence prediction in the wake center and in the free stream are obviously too high, similar to the aforementioned model. The $k-\varepsilon$ model seems to not be able to resolve strong spatial gradients in the distribution of turbulent kinetic energy. Aside from a significantly lower correlation coefficient $R_{\mathrm{k}^{*}, \mathrm{CMR}}=0.378$ than in the other predictions, the geometrical variance $\mathrm{VG}_{\mathrm{k}^{*}, \mathrm{CMR}}=89.922$ is almost 1 order of magnitude higher than in the other predictions.

\subsection{Test case B: high-turbulence uniform inflow}

\subsubsection{Power and thrust predictions}

A second set of power and thrust predictions is compared for inflow conditions of higher turbulence. A turbulence grid installed at the wind tunnel inlet generates a uniform wind field with a turbulence intensity of TI $=10.0 \%$ at the location of the first turbine rotor. For this high background turbulence level, the turbine power and thrust are compared for three turbine separation distances $x / D=2.77,5.18$ and 9.00 (test cases $\mathrm{B}_{1}, \mathrm{~B}_{2}$ and $\mathrm{B}_{3}$ ). The power and thrust predictions for test case $\mathrm{B}$ are compared in Fig. 7a-f. A comparison of the respective numerical values is presented in Table 5 .

Comparing the upstream turbine power curve for high background turbulence (test cases $\mathrm{B}_{2}$, Fig. 7c) to the upstream turbine power curve of low background turbulence (test case A, Fig. 5a) a very similar curve shape is observed. At increased background turbulence, the maximum power coefficient is measured at the same level as for low background turbulence. Furthermore, the runaway tip speed ratio at $\lambda=11.4$, at which the rotor no longer produces energy, is very similar for both inlet turbulence levels. The most noticeable difference is the transition to stall at a tip speed ratio of about $\lambda=3.5$ and lower. For higher background turbulence, the transition into stall is much smoother compared to low inlet turbulence.

The predictions of $C_{\mathrm{P}, \mathrm{T} 1}$ at its design operating point $\lambda_{\mathrm{T} 1}=6.0$ are again very accurate, scattering only about $\pm 7 \%$ around the experimental value. The predictions of the thrust coefficient $C_{\mathrm{T}, \mathrm{T} 1}$ also match very well. As previously observed in test case $\mathrm{A}$, the $C_{\mathrm{T}, \mathrm{T} 1}$ is slightly under predicted, up to $-9 \%$ at the most in this case. Comparing the performance results of the downstream turbine, the best predictions are made for the lowest turbine separation distance $x / D=2.77$ (test case $\mathrm{B}_{1}$, Fig. 7a). The experimentally measured power coefficient $C_{\mathrm{P}, \mathrm{T} 2}$ is well matched, with a total deviation of about $\pm 15 \%$. The downstream turbine thrust coefficient $C_{\mathrm{T}, \mathrm{T} 2}$ is predicted within $\pm 10 \%$ by all the modellers in test case $\mathrm{B}_{1}$. The predictions by $\mathrm{CMR}$ and $\mathrm{CD}$-adapco most closely match the experimental results.

Increasing the turbine separation distance to $x / D=5.18$ in test case $\mathrm{B}_{2}$, the scatter in the results becomes significantly larger (Fig. 7c). The scatter in the downstream turbine power coefficient $C_{\mathrm{P}, \mathrm{T} 2}$ increases to about $\pm 20 \%$ in both directions. The FRR model by LUT results in a very good prediction of the downstream turbine power coefficient, while their ACL model overpredicts the power significantly. This can be directly related to different wake flow predicted by the two models. The wake flow acts as inflow for the downstream turbine (compare Fig. 8a further down). Conversely, UU-DTU's Ellipsys3D calculation underpredicts the downstream turbine performance significantly, even though the wake characteristics are predicted very accurately. Vrije also underpredicts the downstream turbine power significantly. This is rather surprising since the wake deficit at $x / D=5.18$ is slightly underpredicted as well, and more power should be left in the flow for the downstream turbine. The scatter in the thrust calculations, as presented in Fig. $7 \mathrm{~d}$, is in general smaller than for the power predictions for all models, with most simulations underpredicting the experimental value. The thrust coefficient is less sensitive to a correct prediction of the incoming velocity field than the power coefficient. The thrust coefficient is indirectly proportional to the incoming velocity squared $\left(\sim U_{\text {ref }}^{2}\right)$, while the power coefficient is even more sensible to an incorrect prediction of the incoming velocity field $\left(\sim U_{\text {ref }}^{3}\right)$. Surprisingly, LUT's FRR model gives the smallest value for the downstream turbine thrust coefficient, although the power and wake predictions for this downstream distance match the experimental results very well.

With a further increase in turbine separation distance to $x / D=9.00$ (test case $\mathrm{B}_{3}$ ), the experimentally measured downstream turbine power coefficient recovers to $C_{\mathrm{P}, \mathrm{T} 2}=0.270$. The variation in the simulations, as shown in Fig. 7e, is seen to be even bigger for this downstream distance, reaching a scatter of more than $30 \%$. The same trend as already seen for smaller separation distances is observed: UU-DTU's and Vrije's simulations clearly underpredict the power coefficient, while LUT's ACL model considerably overestimates the downstream turbine power. The thrust predictions show similar tendencies to the power predictions but are seen to match the experimentally measured value better (Fig. 7f). 
(a)

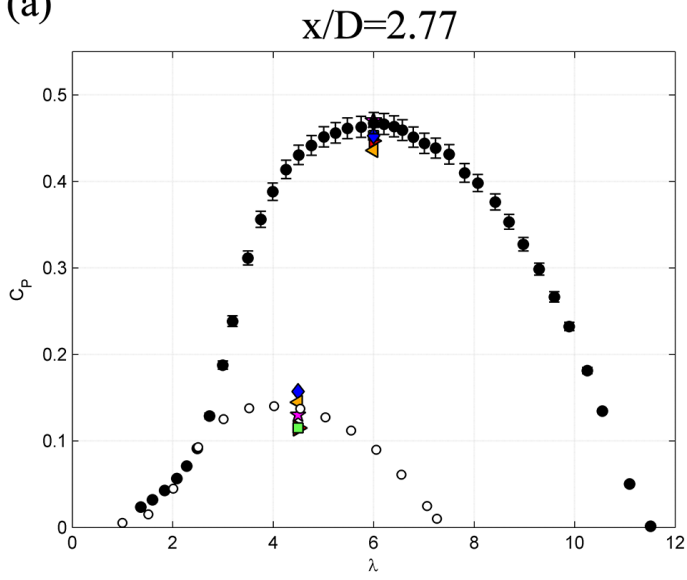

(c)

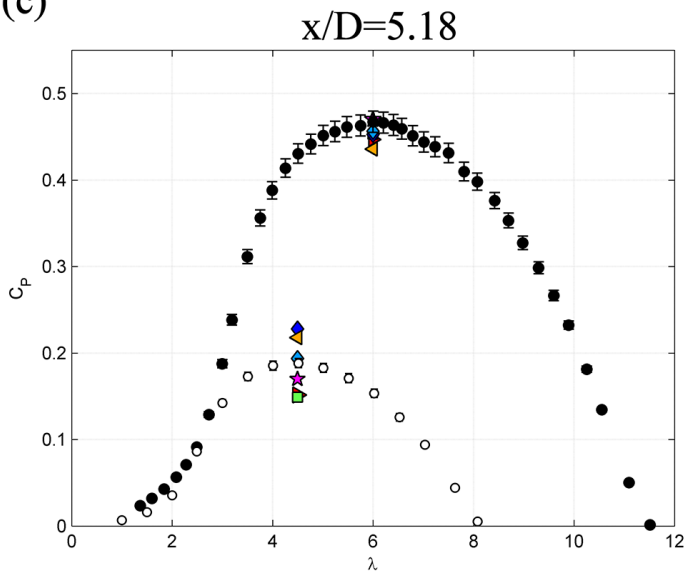

(e)

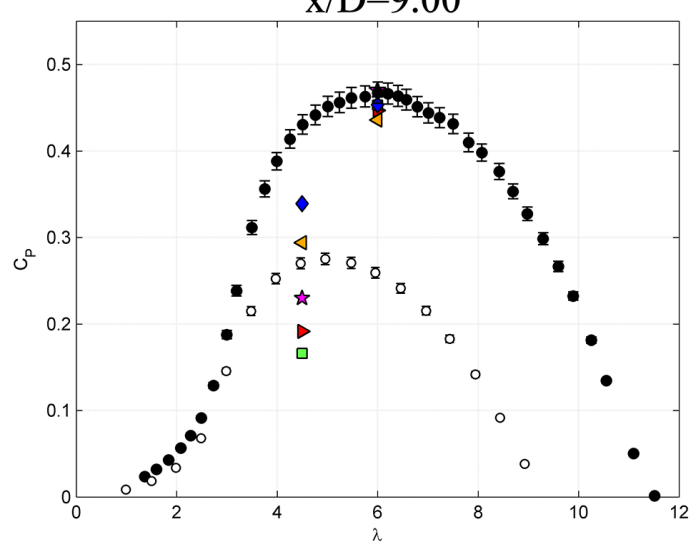

(b)

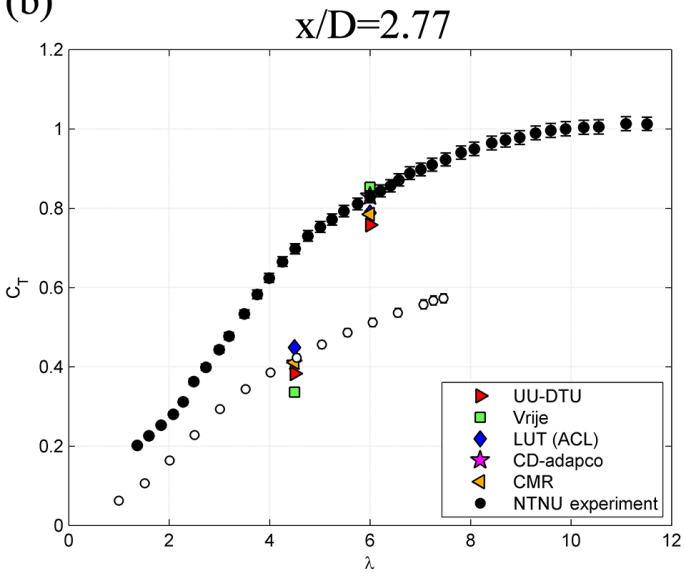

(d)

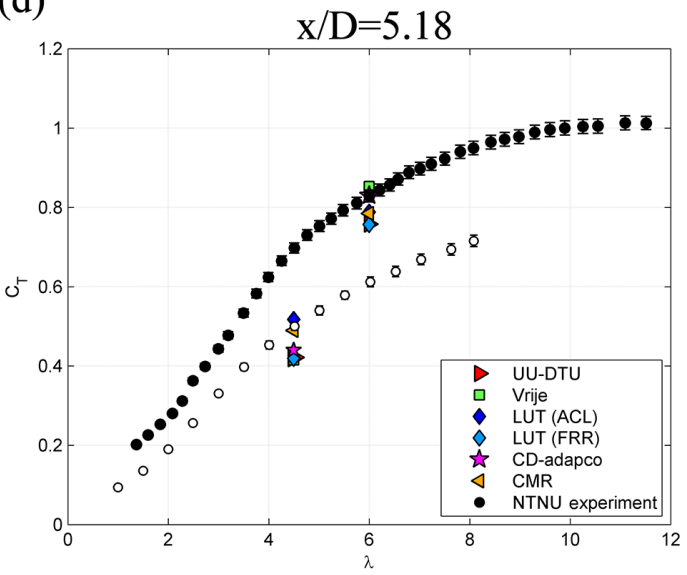

(f)

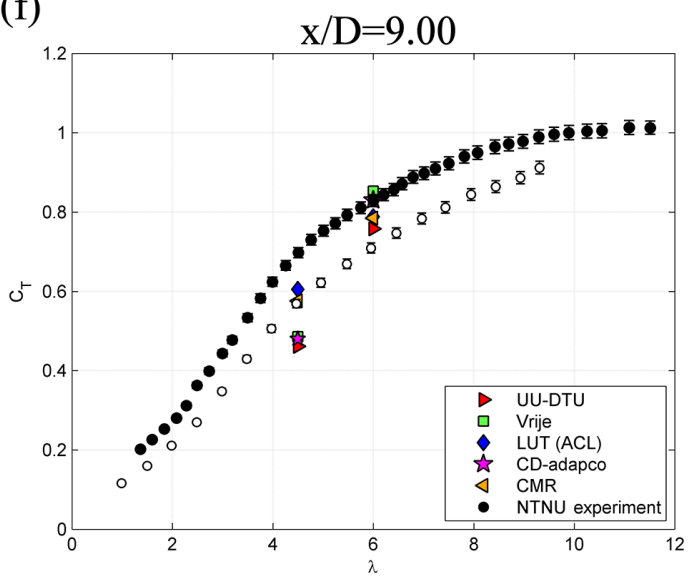

Figure 7. Power coefficient $C_{\mathrm{P}}(\mathbf{a}, \mathbf{c}, \mathbf{e})$ and thrust coefficient $C_{\mathrm{T}}(\mathbf{b}, \mathbf{d}, \mathbf{f})$ for $\mathrm{T} 1$ (filled symbols) and $\mathrm{T} 2$ (empty circles) compared for test cases $\mathrm{B}_{1}, \mathrm{~B}_{2}$ and $\mathrm{B}_{3}$. The downstream turbine $\mathrm{T} 2$ is positioned at $x / D=2.77(\mathbf{a}, \mathbf{b}), 5.18(\mathbf{c}, \mathbf{d})$ and $9.00(\mathbf{e}, \mathbf{f})$ downstream of $\mathrm{T} 1$. The upstream turbine $\mathrm{T} 1$ is operated at $\lambda_{\mathrm{T} 1}=6.0$. The reference velocity is $U_{\mathrm{ref}}=11.5 \mathrm{~m} \mathrm{~s}^{-1}$.

\subsubsection{Wake predictions}

For the high background turbulence test case B, the participants were asked to predict the mean and turbulent wake characteristics at three downstream distances $x / D_{\mathrm{T} 2}=2.77$,
5.18 and 8.50. Note that the horizontal wake profiles were extracted from test case $\mathrm{B}_{3}$, in which the downstream turbine $\mathrm{T} 2$ was installed at $x / D_{\mathrm{T} 2}=9.00$ and operated at $\lambda_{\mathrm{T} 2}=4.5$. The wake flow as measured at $x / D_{\mathrm{T} 2}=8.50$ therefore experienced the induction of the downstream turbine, which 
Table 5. Numerical values of power coefficient $C_{\mathrm{P}}$ and thrust coefficient $C_{\mathrm{T}}$ for test cases $\mathrm{B}_{1}, \mathrm{~B}_{2}$ and $\mathrm{B}_{3}$. The downstream turbine $\mathrm{T} 2$ is positioned at $2.77 \mathrm{D}\left(\mathrm{B}_{1}\right), 5.18 \mathrm{D}\left(\mathrm{B}_{2}\right)$ and $9.00 \mathrm{D}\left(\mathrm{B}_{3}\right)$ downstream of $\mathrm{T} 1$. $\mathrm{T} 1$ is operated at $\lambda_{\mathrm{T} 1}=6.0$ and $\mathrm{T} 2$ is operated at $\lambda_{\mathrm{T} 1}=4.5$, referring to the reference velocity $U_{\text {ref }}=11.5 \mathrm{~m} \mathrm{~s}^{-1}$.

\begin{tabular}{|c|c|c|c|c|c|c|c|c|c|}
\hline & & \multicolumn{2}{|c|}{$\begin{array}{l}\text { Upstream turbine } \\
\mathrm{T} 1\end{array}$} & \multicolumn{2}{|c|}{$\begin{array}{l}\text { Downstream turbine } \\
\text { T2 at } 2.77 D\left(\mathrm{~B}_{1}\right)\end{array}$} & \multicolumn{2}{|c|}{$\begin{array}{l}\text { Downstream turbine } \\
\text { T2 at } 5.18 D\left(\mathrm{~B}_{2}\right)\end{array}$} & \multicolumn{2}{|c|}{$\begin{array}{l}\text { Downstream turbine } \\
\text { T2 at } 9.00 D\left(\mathrm{~B}_{3}\right)\end{array}$} \\
\hline & & $C_{\mathrm{P}, \mathrm{T} 1}$ & $C_{\mathrm{T}, \mathrm{T} 1}$ & $C_{\mathrm{P}, \mathrm{T} 2}$ & $C_{\mathrm{T}, \mathrm{T} 2}$ & $C_{\mathrm{P}, \mathrm{T} 2}$ & $C_{\mathrm{T}, \mathrm{T} 2}$ & $C_{\mathrm{P}, \mathrm{T} 2}$ & $C_{\mathrm{T}, \mathrm{T} 2}$ \\
\hline UU-DTU & 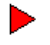 & 0.447 & 0.758 & 0.115 & 0.383 & 0.152 & 0.423 & 0.192 & 0.462 \\
\hline Vrije & $\square$ & 0.453 & 0.853 & 0.115 & 0.336 & 0.149 & 0.415 & 0.166 & 0.486 \\
\hline LUT (ACL) & & 0.453 & 0.788 & 0.157 & 0.449 & 0.228 & 0.518 & 0.339 & 0.605 \\
\hline LUT (FRR) & & 0.456 & 0.756 & - & - & 0.194 & 0.419 & - & - \\
\hline CD-adapco & 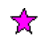 & 0.470 & 0.830 & 0.130 & 0.410 & 0.170 & 0.440 & 0.230 & 0.480 \\
\hline CMR & $\triangleleft$ & 0.436 & 0.785 & 0.145 & 0.411 & 0.218 & 0.490 & 0.294 & 0.576 \\
\hline Experiment & $\bullet$ & 0.468 & 0.833 & 0.137 & 0.423 & 0.188 & 0.500 & 0.270 & 0.569 \\
\hline
\end{tabular}

Table 6. Statistical performance measures FB, NMSE, MG, VG and $R$ of the normalized mean velocity $U^{*}$ and normalized turbulent kinetic energy $k^{*}$ predictions of the five different models for test case B3. The wake flow is predicted at stream-wise measurement positions $x / D=2.77,5.18$ and 8.50 downstream of $\mathrm{T} 1$.

\begin{tabular}{|c|c|c|c|c|c|c|c|c|c|c|c|c|}
\hline & & & $\mathrm{FB}_{U^{*}}$ & $\mathrm{NMSE}_{U^{*}}$ & $\mathrm{MG}_{U^{*}}$ & $\mathrm{VG}_{U^{*}}$ & $R_{U^{*}}$ & $\mathrm{FB}_{\mathrm{k}^{*}}$ & $\mathrm{NMSE}_{\mathrm{k}^{*}}$ & $\mathrm{MG}_{\mathrm{k}^{*}}$ & $\mathrm{VG}_{\mathrm{k}^{*}}$ & $R_{\mathrm{k}^{*}}$ \\
\hline \multirow{6}{*}{ 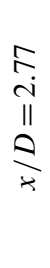 } & UU-DTU & $D$ & 0.027 & 0.001 & 1.025 & 1.002 & 0.968 & -0.329 & 0.111 & 0.671 & 1.219 & 0.911 \\
\hline & Vrije & $\square$ & 0.003 & 0.000 & 1.005 & 1.002 & 0.959 & 0.222 & 0.050 & 1.239 & 1.847 & -0.008 \\
\hline & LUT (ACL) & & -0.013 & 0.000 & 0.981 & 1.013 & 0.845 & 0.055 & 0.003 & 1.048 & 1.243 & 0.468 \\
\hline & LUT (FRR) & & -0.009 & 0.000 & 0.988 & 1.003 & 0.949 & 0.525 & 0.296 & 1.771 & 1.539 & 0.720 \\
\hline & CD-adapco & h & 0.048 & 0.002 & 1.060 & 1.006 & 0.970 & -0.007 & 0.000 & 1.035 & 1.057 & 0.912 \\
\hline & CMR & $\Delta$ & -0.014 & 0.000 & 0.982 & 1.007 & 0.913 & -0.771 & 0.698 & 0.404 & 2.720 & 0.417 \\
\hline \multirow{5}{*}{ 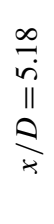 } & UU-DTU & & 0.021 & 0.000 & 1.017 & 1.002 & 0.964 & -0.203 & 0.041 & 0.794 & 1.124 & 0.850 \\
\hline & Vrije & $\square$ & 0.020 & 0.000 & 1.024 & 1.003 & 0.957 & 0.047 & 0.002 & 0.988 & 1.361 & 0.371 \\
\hline & LUT (ACL) & & -0.035 & 0.001 & 0.954 & 1.012 & 0.929 & 0.423 & 0.188 & 1.459 & 1.405 & 0.273 \\
\hline & CD-adapco & $\underline{x}$ & 0.054 & 0.003 & 1.065 & 1.007 & 0.971 & -0.128 & 0.017 & 0.942 & 1.059 & 0.934 \\
\hline & CMR & 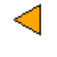 & -0.030 & 0.001 & 0.963 & 1.005 & 0.937 & -0.598 & 0.393 & 0.483 & 1.980 & 0.705 \\
\hline \multirow{5}{*}{$\begin{array}{l}0 \\
n \\
\infty \\
\| \\
\vdots \\
\vdots\end{array}$} & UU-DTU & 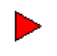 & 0.028 & 0.001 & 1.029 & 1.001 & 0.970 & -0.059 & 0.004 & 0.964 & 1.052 & 0.812 \\
\hline & Vrije & $\square$ & 0.062 & 0.004 & 1.078 & 1.014 & 0.958 & -0.159 & 0.026 & 0.830 & 1.112 & 0.656 \\
\hline & LUT (ACL) & & 0.018 & 0.000 & 1.015 & 1.001 & 0.936 & 0.706 & 0.569 & 2.095 & 1.828 & 0.594 \\
\hline & CD-adapco & 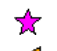 & 0.116 & 0.013 & 1.143 & 1.032 & 0.962 & 0.166 & 0.028 & 1.259 & 1.130 & 0.811 \\
\hline & CMR & $<$ & -0.040 & 0.002 & 0.957 & 1.004 & 0.955 & -0.465 & 0.228 & 0.596 & 1.410 & 0.804 \\
\hline
\end{tabular}

was located only $x / D_{\mathrm{T} 2}=0.50$ further downstream. The horizontal wake profiles of the normalized mean velocity $U / U_{\text {ref }}$ and normalized turbulent kinetic energy $k^{*}=k / U_{\text {ref }}^{2}$ are compared in Fig. 8a-f.

The wake characteristics of the flow $x / D_{\mathrm{T} 2}=2.77$ downstream of T1 are presented in Fig. 8a and b. For this case, LUT simulated the wake flow with two different models, the simpler ACL model and the computationally more expensive FRR model. At this downstream distance the mean wake profiles are characterized by two distinct minima. The experimental results clearly show that a Gaussian wake shape has not yet developed. A very accurate prediction of the mean wake shape is given by UU-DTU's simulation, but the CD- adapco model and the FRR model by LTU also capture the shape very well. LTU's ACL model, however, only predicts one distinct minimum in the mean wake profile. Only one minimum is also predicted by CMR, while the mean velocity profile is rather skewed. Vrije's simulations match the experimental measurements significantly better for a higher background turbulence level than for the lower turbulence level of test case A, predicting both the level and wake shape fairly well.

The fact that all predictions approximated the level of mean velocity deficit fairly well is also reflected in the statistical performance measures as presented in Table 6 (upper left section). $\mathrm{FB}_{U^{*}}$ and $\mathrm{NMSE}_{U^{*}}$ are close to zero, while 
(a)

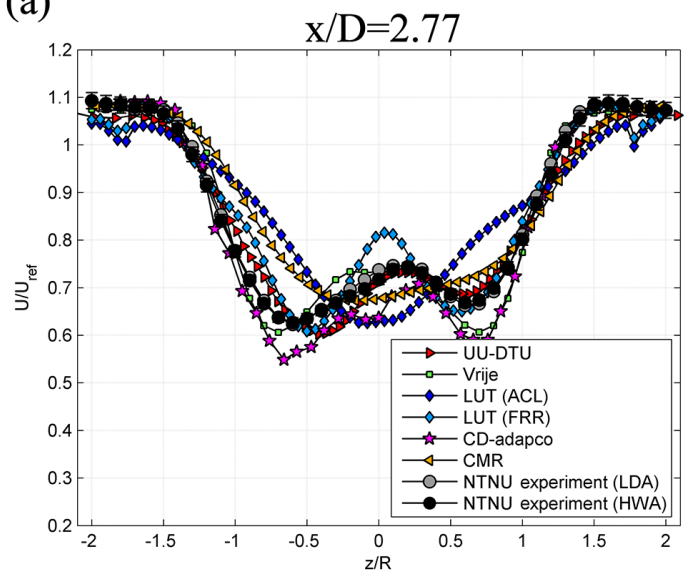

(c)

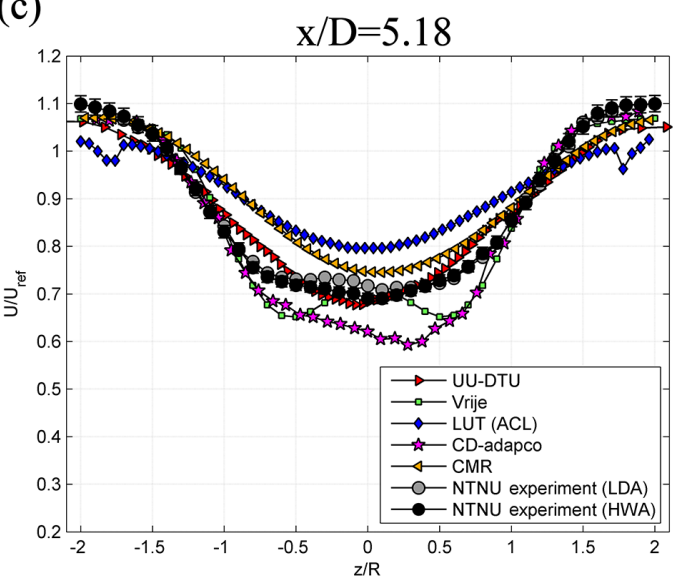

(e)

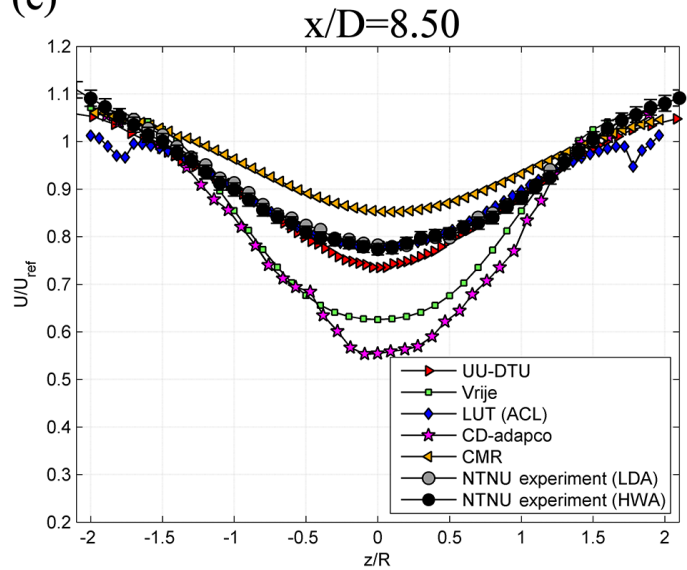

(b)

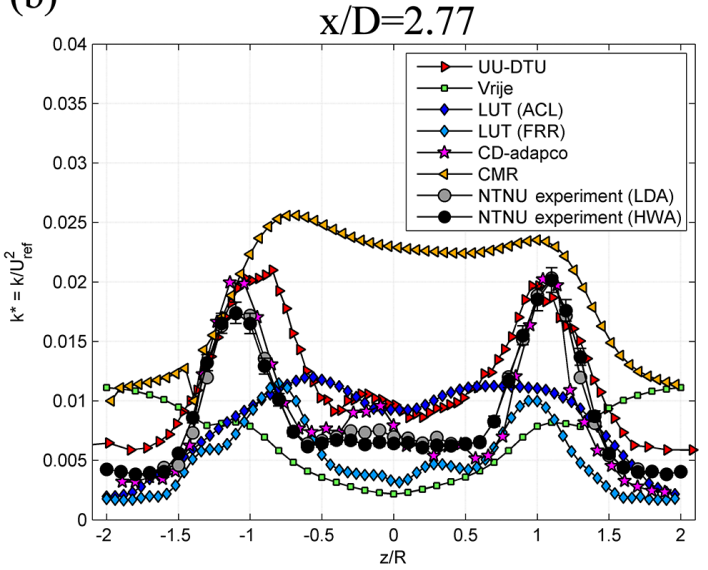

(d)

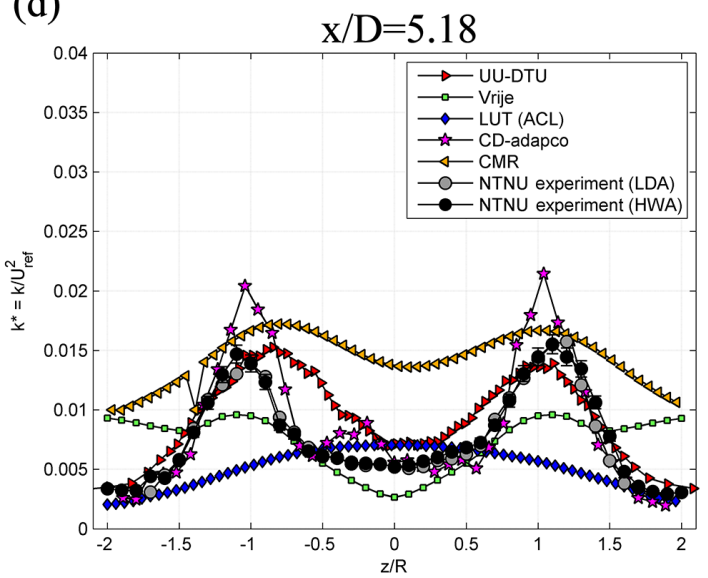

(f)

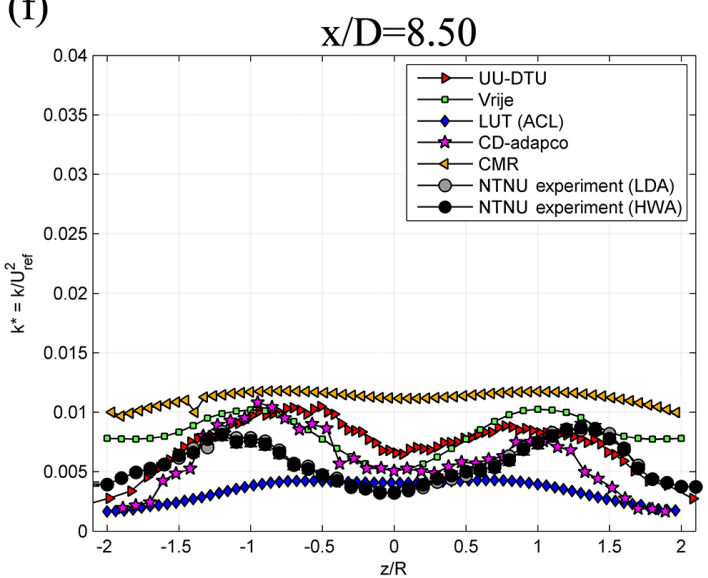

Figure 8. Normalized mean velocity $U / U_{\text {ref }}(\mathbf{a}, \mathbf{c}, \mathbf{e})$ and normalized turbulent kinetic energy $k / U_{\text {ref }}^{2}(\mathbf{b}, \mathbf{d}, \mathbf{f})$ in the wake $x / D=2.77(\mathbf{a}, \mathbf{b})$, $5.18(\mathbf{c}, \mathbf{d})$ and $8.50(\mathbf{e}, \mathbf{f})$ behind $\mathrm{T} 1$ for test case setup $\mathrm{B}_{3}$. The upstream turbine $\mathrm{T} 1$ is operated at $\lambda_{\mathrm{T} 1}=6.0$. The reference velocity is $U_{\text {ref }}=11.5 \mathrm{~m} \mathrm{~s}^{-1}$.

$\mathrm{MG}_{U^{*}}$ and $\mathrm{VG}_{U^{*}}$ show only very small deviations from the perfect correlation value 1 for all predictions. The highest correlation coefficient $R_{U^{*}}$ is reached by CD-adapco with 0.970 , closely followed by UU-DTU, Vrije and the FRR model by LUT. The correlation coefficient of CMR's pre- diction is a few percent lower, while LUT's ACL model that only predicts one minimum scores lowest.

Very good predictions of the distribution of the turbulent kinetic energy are presented by CD-adapco as well as UUDTU. Both simulations predict the magnitude and location of 
the two peaks around $z / R= \pm 1$ as well as the region of lower turbulence in the center of the wake very accurately. This is also reflected in the high values of the correlation coefficient $R_{\mathrm{k}^{*} \text {,CD-adapco }}=0.912$ and $R_{\mathrm{k}^{*} \text {,UU-DTU }}=0.911$ as shown in the upper left section of Table 6. LUT's FRR simulation manages to reproduce the general shape of the turbulence profile, but the levels are about $50 \%$ below the measured turbulence values, resulting in a significantly lower correlation coefficient $R_{\mathrm{k}^{*} \text {,LUT(FRR) }}=0.720$. Similar levels are observed for LUT's ACL simulation, which additionally smears out the turbulence to the center of the wake, giving a correlation coefficient of $R_{\mathrm{k}^{*}, \mathrm{LUT}(\mathrm{ACL})}=0.468$. It has been discussed that the tip-loss correction model included in the ACL model could have contributed to killing the turbulent peaks. Vrije's model based on a standard $k-\omega$ turbulence model underpredicts the peaks in the shear layer significantly; they are observed to be lower than the turbulence levels in the free stream flow, which are overpredicted by more than 1 order of magnitude. A very low and negative correlation coefficient of $R_{\mathrm{k}^{*}, \text { Vrije }}=-0.008$ confirms this observation. The negative sign stems from a mainly negative correlation, meaning that turbulence levels are predicted to decrease from the free stream to the shear layer, while they actually increase in the experimentally measured profile. CMR's simulations predict too-high turbulence levels at the peaks, but surprisingly also in the wake center and in the unaffected free stream flow. A rather low correlation of $R_{\mathrm{k}^{*}, \mathrm{CMR}}=0.417$ with the experimental data is achieved, while the normalized mean squared error $\mathrm{NMSE}_{\mathrm{k}^{*}, \mathrm{CMR}}=0.698$ is significantly higher than for the other predictions. A possible reason for that blurry turbulence distribution could be the $k-\varepsilon$ turbulence model used.

Moving downstream to $x / D_{\mathrm{T} 2}=5.18$, a more Gaussian mean velocity profile with only one distinct minimum develops, as shown in Fig. 8c. The general shape of the mean velocity profile is in this case well predicted by almost all the simulations; only Vrije's simulation indicates a near-wake shape with two minima, but it still results in fairly good statistical performance measures, as presented in the middle left section of Table 6. Again, UU-DTU's model gives a very good match with the experimentally measured profiles, which is also reflected in very low $\mathrm{FB}_{U^{*}}$ and $\mathrm{NMSE}_{U^{*}}$ values. $\mathrm{MG}_{U^{*}}$ and $\mathrm{VG}_{U^{*}}$ approach the perfect value 1 very closely and a very high correlation coefficient of $R_{U^{*} \text {,UU-DTU }}=0.964$ is calculated. CMR's model computes a slightly asymmetric mean wake profile, somewhat underpredicting the velocity deficit, but still performing well overall, as indicated in the correlation coefficient of $R_{U^{*}, \mathrm{CMR}}=0.937$. LUT modeled the $5.18 \mathrm{D}$ wake using their simpler ACL model, which considerably underpredicts the mean velocity deficit. The statistical performance measures are therefore slightly poorer than for the other predictions for this case, as shown in Table 6. CD-adapco's IDDES simulation overpredicts the mean wake velocity deficit to some extent, but still reaches the highest correlation coefficient $R_{U^{*}, \mathrm{CD} \text {-adapco }}=0.971$. This might be due to the almost perfect correlation of the flow in the free stream and shear layer, although the mean velocities in the wake center are predicted somewhat lower than measured in the experiment.

The turbulence profiles for $x / D_{\mathrm{T} 2}=5.18$ as presented in Fig. 8d show a similar picture as seen earlier for $x / D_{\mathrm{T} 2}=2.77$. The best predictions are made by CDadapco's IDDES computation and UU-DTU's LES simulation, with both predictions resulting in very low error indicators $\mathrm{FB}_{\mathrm{k}}$ 的 $\mathrm{NMSE}_{\mathrm{k}^{*}}$. A very high correlation coefficient $R_{\mathrm{k}^{*} \text {,CD-adapco }}=0.934$ to the experimental dataset is achieved by CD-adapco's prediction, although the turbulence peaks in the tip vortex region at $z / R= \pm 1.0$ are somewhat overpredicted. The magnitude of the peaks in the shear layer is almost perfectly predicted by UU-DTU's computation. However, compared to the experimental dataset, the peaks are too broad, which overpredicts the TKE in the wake center. This is reflected in a fairly good but somewhat lower correlation coefficient of $R_{\mathrm{k}^{*} \text {,UU-DTU }}=0.850$. Too-smooth turbulence profiles are predicted by CMR as well as LUT's ACL model, clearly overpredicting $\left(\mathrm{MG}_{\mathrm{k}^{*}, \mathrm{CMR}}=0.483\right)$ or underpredicting $\left(\mathrm{MG}_{\mathrm{k}^{*}, \mathrm{LUT}}=1.495\right)$ the mean turbulence levels. Vrije's turbulence prediction is very similar to the profile measured at $x / D=2.77$ and again results in a rather low correlation coefficient of $R_{\mathrm{K}^{*} \text {, Vrije }}=0.371$.

A challenging test case is shown for the wake measured at downstream position $x / D_{\mathrm{T} 2}=8.50$, only half a rotor diameter upstream of the rotor of T2 (Fig. 8e and f). A smooth Gaussian mean velocity profile developed, while velocity deficit further decreased. Again, UU-DTU predicted the mean wake well, scoring the highest in the correlation coefficient $R_{U^{*}, \mathrm{UU}-\mathrm{DTU}}=0.970$, as shown in the lower left section of Table 6. Although the mean profile predicted from LUT's ACL model matches the experimental values very well for this case, it is very similar to the profile that was already predicted for $5.18 \mathrm{D}$, where it clearly underpredicted the velocity deficit. Very low error values of $\mathrm{FB}_{U^{*}, \text { LUT }}$ and $\mathrm{NMSE}_{U^{*}, \text { LUT }}$ are computed, while $\mathrm{MG}_{U^{*}, \text { LUT }}$ and $\mathrm{VG}_{U^{*}, \text { LUT }}$ are close to 1 . The correlation coefficient $R_{U^{*} \text {,LUT }}=0.936$ is fairly good, but scores slightly lower than the other predictions. This might be due to obvious discontinuities of the mean velocity profile at $z / R= \pm 1.7$. CDadapco's simulation strongly overpredicts the mean velocity deficit in the wake at this downstream distance. Surprisingly, the mean velocity deficit even grows noticeably in comparison to the mean wake profile predicted at $5.18 \mathrm{D}$. As shown in the numbers in the lower left section of Table 6 , this obvious deviation is also resembled in significantly higher deviations of the mean geometrical bias $\mathrm{MG}_{U^{*}}$ and geometrical variance $\mathrm{VG}_{U^{*}}$ compared with the corresponding values of the other predictions. Vrije's simulation also overpredicts the mean velocity deficit for this case. Correspondingly, $\mathrm{MG}_{U^{*}}$ and $\mathrm{VG}_{U^{*}}$ give the second highest deviation from the experimentally measured profile. Remarkably, the averaged velocity deficit at $8.50 \mathrm{D}$ did not recover very much from the one predicted at $5.18 \mathrm{D}$. As already observed for smaller down- 
stream distances, CMR also predicts a slightly too low velocity deficit for $8.50 \mathrm{D}$. Almost all statistical performance measures for CMR, however, are significantly better at this further wake distance than at the closer measurement stations.

Analyzing the turbulence profile as shown in Fig. 8f, the tip vortex peaks decay to about $50 \%$ of the magnitude measured at $5.18 \mathrm{D}$. Both CD-adapco's IDDES simulation and UU-DTU's LES simulation give a fairly good approximation of the turbulence profile, as reflected in the highest correlation coefficients $R_{\mathrm{k}^{*}, \mathrm{CD} \text {-adapco }}=0.811$ and $R_{\mathrm{k}^{*} \text {,UU-DTU }}=0.812$. Because the decay of the turbulence in the wake center is slightly underpredicted by both simulations, these values do not score as high as for the near-wake measurement stations. CMR overpredicts the turbulence levels at $8.50 \mathrm{D}$, smearing out the turbulence profile to an almost constant line. The acceptably good correlation coefficient $R_{\mathrm{k}^{*} \text {,CMR }}=0.804$ gives a wrong impression in this case because $R$ is insensitive to addition, as introductorily stated in Sect. 2.5.2, and the profile is basically shifted upwards. The high deviations from 1.00 in $\mathrm{MG}_{\mathrm{k}^{*}, \mathrm{CMR}}$ and $\mathrm{VG}_{\mathrm{k}^{*}, \mathrm{CMR}}$, however, indicate the significant mismatch.

Conversely, LUT's ACL model underpredicts the turbulence considerably. Higher deviations in $\mathrm{MG}_{\mathrm{k}^{*} \text {,LUT }}$ and $\mathrm{VG}_{\mathrm{K}^{*} \text {,LUT }}$ are observed compared to for the other predictions. The turbulence levels predicted by Vrije's $k-\omega$ model at $8.50 \mathrm{D}$ are observed to be very similar to those already predicted at lower separation distances. This indicates that the turbulent decay rate is not well captured for this case. Compared the lower separation distances, the predicted TKE profile matches better with the measured profile, resulting in acceptable statistical performance measures (e.g., $\left.R_{\mathrm{k}^{*}, \text { Vrije }}=0.656\right)$.

\subsection{Test case C: high-turbulence nonuniform shear flow}

\subsubsection{Power and thrust predictions}

For the last test case the complexity of the inflow conditions is increased. The inflow to the test section is no longer spatially uniform. Another custom-made grid with vertically increasing distance between the horizontal bars is placed at the test section inlet, generating a shear flow that can be approximated by the power law exponent $\alpha=0.11$. The background turbulence of this grid is measured to be $\mathrm{TI}=10.1 \%$ over the rotor area at the location of the first turbine rotor. This makes the effects of shear flow well comparable to test case B since basically the same background turbulence level is predominating. For test case $\mathrm{C}$ the turbine power and thrust are compared only for one turbine separation distance, $x / D_{\mathrm{T} 2}=5.18$. The power and thrust predictions for the shear flow test case are presented in Fig. 9, while the exact numerical values are shown in Table 7.

Comparing the upstream turbine power curve of test case $\mathrm{C}$ (Fig. 9a) to the upstream turbine power curve of uniform inflow test case B (Fig. 7c), a very similar curve shape is observed. Taking a closer look, however, a slightly lower maximum power coefficient is measured in case $\mathrm{C}$ and a marginally earlier run-away point is found at $\lambda=11.2$. This is assumed to stem from the fact that the reference velocity $U_{\text {ref }}$ for this test case is defined at the center of the rotor at hub height. Due to the vertically nonlinear gradient in velocity distribution (see Fig. 4), the rotor equivalent wind speed (Wagner et al., 2014) is found to be slightly higher than $U_{\text {ref }}$ measured at hub height. Therefore, the $C_{\mathrm{P}}$ and $C_{\mathrm{T}}$ calculations that are a priori defined to refer to the hub height reference wind speed $U_{\text {ref }}=11.5 \mathrm{~m} \mathrm{~s}^{-1}$ are slightly lower for test case $\mathrm{C}$ than for test case $\mathrm{B}$. The rotor-swept area is exposed to the same kinetic energy in cases B and C. The wind speed at the predefined reference height in test case $\mathrm{C}$, however, does represent the rotor-averaged wind speed (for a more detailed investigation the reader is referred to Wagner et al., 2014).

The predictions of $C_{\mathrm{P}, \mathrm{T} 1}$ at the turbine design operating point $\lambda_{\mathrm{T} 1}=6.0$ are again very precise, showing a scatter of less than $\pm 5 \%$ from the measured value. All the contributions predict a little lower $C_{\mathrm{P}, \mathrm{T} 1}$ value than in test case $\mathrm{B}$, confirming the tendency measured in the experiment. All the predictions of the thrust coefficient $C_{\mathrm{T}, \mathrm{T} 1}$ also give a very good match with the experiment. In this case the spread is about $\pm 5 \%$, which is just slightly outside the measurement uncertainty.

Analyzing the performance results of the downstream turbine at $x / D_{\mathrm{T} 2}=5.18$, the predictions are very good. The scatter in $C_{\mathrm{P}, \mathrm{T} 2}$ is within $\pm 7 \%$, except from UU-DTU's prediction, which is about $24 \%$ lower than the experimental value. This seems to be a systematic deviation because significantly low values have been observed in test cases B already. The predictions of the thrust coefficient are very close to each other; however, they are up to $16 \%$ lower than the measured value at $\lambda_{\mathrm{T} 2}=4.5$. A general tendency to underpredict the thrust is again seen for all test cases $(\mathrm{A}, \mathrm{B}, \mathrm{C})$, but the predictions are significantly closer compared to previous blind test comparisons.

\subsubsection{Wake predictions}

One single wake profile behind the upstream turbine is compared for test case $\mathrm{C}$, in which the turbine is exposed to highly turbulent shear flow at the test section inlet. The mean and turbulent wake characteristics at $x / D_{\mathrm{T} 2}=2.77$ behind the upstream turbine are compared in Fig. 10.

The mean velocity profile (Fig. 10a) has a very similar shape to the wake behind the same turbine exposed to uniform inflow of the same turbulence intensity (Fig. 8a). The mean velocity profile for shear inflow is also characterized by two distinct minima and a smooth transition from the wake to the free stream. Taking a closer look, the wake in case $\mathrm{C}$ is slightly skewed compared to the one measured in test case $\mathrm{B}$. Especially the minimum velocity peak at $z / R \approx-0.7$ is somewhat lower compared to in test case B. It is assumed that low kinetic energy fluid that encounters the lower half 
(a)

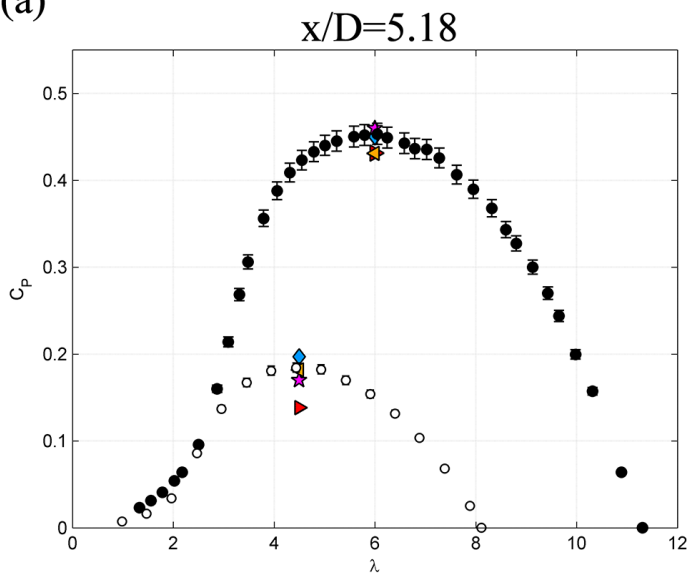

(b)

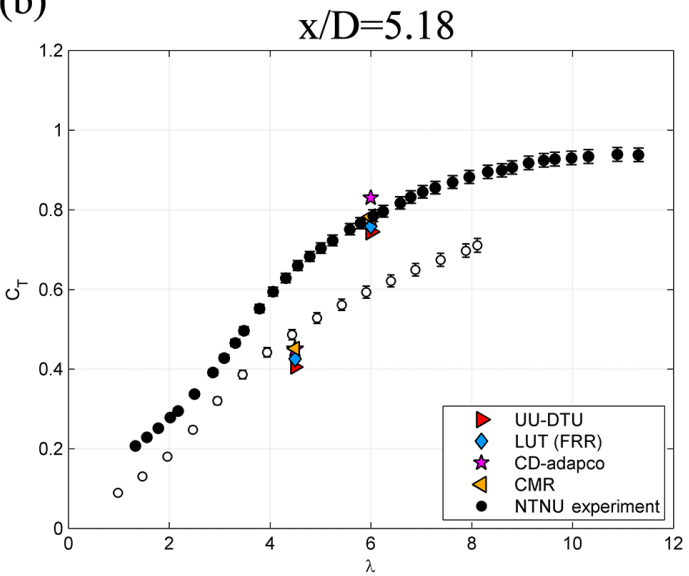

Figure 9. Power coefficient $C_{\mathrm{P}}$ (a) and thrust coefficient $C_{\mathrm{T}}$ (b) for T1 (filled symbols) and T2 (empty circles) compared for test case C. The downstream turbine $\mathrm{T} 2$ is positioned at $x / D=5.18$ downstream of $\mathrm{T} 1$, and the upstream turbine $\mathrm{T} 1$ is operated at $\lambda \mathrm{T} 1=6.0$. The reference velocity $U_{\text {ref }}=11.5 \mathrm{~m} \mathrm{~s}^{-1}$ is the velocity experienced by $\mathrm{T} 1$ at hub height.

Table 7. Numerical values of power coefficient $C_{\mathrm{P}}$ and thrust coefficient $C_{\mathrm{T}}$ for test case $\mathrm{C}$. The downstream turbine T2 is positioned at $5.18 D$ downstream of $\mathrm{T} 1$. T1 is operated at $\lambda_{\mathrm{T} 1}=6.0$ and $\mathrm{T} 2$ is operated at $\lambda_{\mathrm{T} 1}=4.5$, referring to the reference velocity $U_{\text {ref }}=11.5 \mathrm{~m} \mathrm{~s}{ }^{-1}$ measured at hub height.

\begin{tabular}{lllrlr}
\hline & & \multicolumn{2}{c}{ Upstream turbine T1 } & \multicolumn{2}{c}{ Downstream turbine T2 } \\
& & $C_{\mathrm{P}, \mathrm{T} 1}$ & $C_{\mathrm{T}, \mathrm{T} 1}$ & $C_{\mathrm{P}, \mathrm{T} 2}$ & $C_{\mathrm{T}, \mathrm{T} 2}$ \\
\hline UU-DTU & & 0.432 & 0.745 & 0.139 & 0.405 \\
LUT (FRR) & $\bullet$ & 0.451 & 0.758 & 0.197 & 0.426 \\
CD-adapco & $\star$ & 0.460 & 0.830 & 0.170 & 0.450 \\
CMR & $\ngtr$ & 0.431 & 0.782 & 0.182 & 0.452 \\
Experiment & $\bullet$ & 0.453 & 0.785 & 0.184 & 0.486 \\
\hline
\end{tabular}

of the rotor is transported into the measurement plane by the rotation in the wake. Turbulent mixing processes have most likely already evened out this effect at $x / D=2.77$, yet a small difference is detectable.

Four different predictions are compared since Vrije did not simulate test case C. As observed for the earlier test cases, UU-DTU's LES simulation predicts the mean wake shape very accurately. The levels of the two minima are matched very closely, which is also reflected in a high correlation coefficient of $R_{U^{*}, \text { UU-DTU }}=0.965$, as presented in Table 8 . LUT's fully resolved rotor simulation gives a good agreement as well $\left(R_{U^{*}, \text { LUT }}=0.952\right)$; however, the skew in the wake is not as distinct as in the measured profile. CD-adapco predicts the skewed shape of the wake very well, as indicated in the highest correlation coefficient $R_{U^{*}, \mathrm{CD} \text {-adapco }}=0.972$ for this test case; however, the kinetic energy deficit is again slightly too high in the blade tip regions, which is reflected by slightly higher deviations in the fractional bias $\mathrm{FB}_{U^{*}}$ and geometrical mean bias $\mathrm{MG}_{U^{*}}$. As previously observed for test case $\mathrm{B}$, the two mean velocity minima are combined into one in CMR's simulations. Nevertheless, the simulations predict skew in the mean wake profile when compared to CMR's mean wake prediction for test case B. The correlation coefficient $R_{U^{*}, \mathrm{CMR}}=0.898$ is therefore slightly lower than for the other predictions, but it indicates good performance overall.

Analyzing the turbulent kinetic energy profiles for test case $\mathrm{C}$ (Fig. 10b), obvious similarities to those of test case B (Fig. 8b) are observed. UU-DTU's simulations match the experimental results very accurately in the center and the tip region, whereas the turbulence level in the free stream is slightly too high. A similar correlation coefficient $R_{\mathrm{k}^{*} \text {,UU-DTU }}=0.866$ as for test case B is computed. LUT's FRR simulations underpredict one peak significantly, while the turbulence level in the free stream is significantly higher than in the measurements. This is also reflected in a poorer correlation with the experimental data since a correlation coefficient of $R_{\mathrm{k}^{*} \text {,LUT }}=0.666$ is achieved. The TKE predictions by CD-adapco are a very close match to the experimental values for this case. The turbulence peaks in the shear layer as well as the free stream level match the measured profiles very well, while the levels in the wake center are in- 
(a)

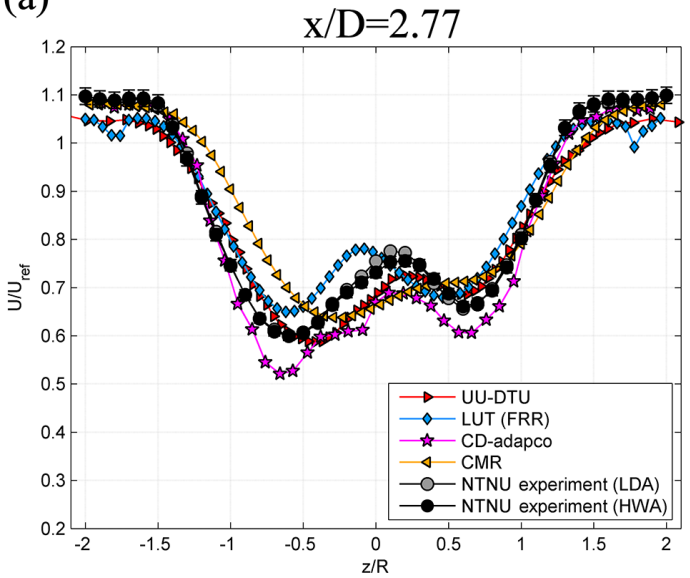

(b)

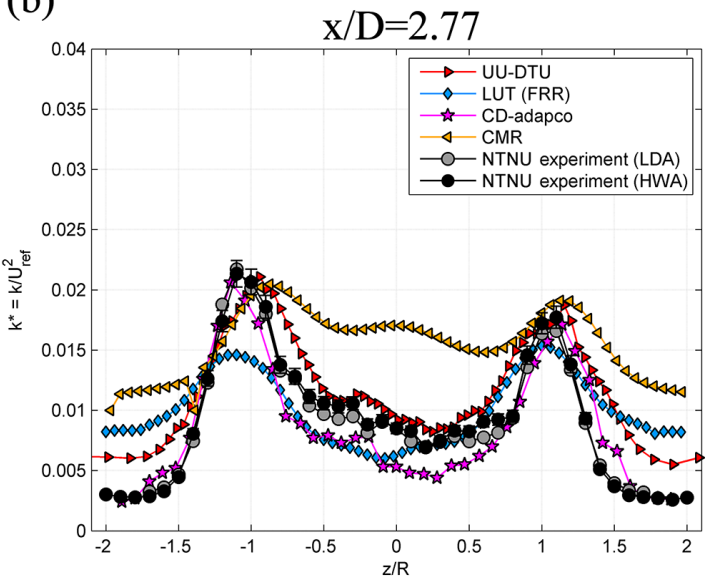

Figure 10. Normalized mean velocity $U / U_{\text {ref }}$ (a) and normalized turbulent kinetic energy $k / U_{\text {ref }}(\mathbf{b})$ in the wake $x / D=2.77$ behind $\mathrm{T} 1$ measured for test case $\mathrm{C}$. The upstream turbine $\mathrm{T} 1$ is operated at $\lambda_{\mathrm{T} 1}=6.0$. The reference velocity $U_{\text {ref }}=11.5 \mathrm{~m} \mathrm{~s}{ }^{-1}$ is the velocity experienced by $\mathrm{T} 1$ at hub height.

Table 8. Statistical performance measures FB, NMSE, MG, VG and $R$ of the normalized mean velocity $U^{*}$ and normalized turbulent kinetic energy $k^{*}$ predictions of the four different models for test case $\mathrm{C}$. The wake flow is predicted at stream-wise measurement position $x / D=2.77$ downstream of $\mathrm{T} 1$.

\begin{tabular}{|c|c|c|c|c|c|c|c|c|c|c|c|}
\hline & & $\mathrm{FB}_{U^{*}}$ & $\mathrm{NMSE}_{U^{*}}$ & $\mathrm{MG}_{U^{*}}$ & $\mathrm{VG}_{U^{*}}$ & $R_{U^{*}}$ & $\mathrm{FB}_{\mathrm{k}} *$ & $\mathrm{NMSE}_{\mathrm{k}} *$ & $\mathrm{MG}_{\mathrm{k}^{*}}$ & $\mathrm{VG}_{\mathrm{k}^{*}}$ & $R_{\mathrm{k}^{*}}$ \\
\hline UU-DTU & $D$ & 0.042 & 0.002 & 1.038 & 1.003 & 0.965 & -0.246 & 0.061 & 0.684 & 1.353 & 0.866 \\
\hline & 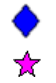 & $\begin{array}{r}-0.005 \\
0.061\end{array}$ & & & & & & & & & $\begin{array}{l}0.666 \\
0.795\end{array}$ \\
\hline CMR & $\triangleleft$ & -0.002 & 0.000 & 0.993 & 1.009 & 0.898 & -0.517 & 0.286 & 0.493 & 2.161 & 0.742 \\
\hline
\end{tabular}

significantly underpredicted. The resulting correlation coefficient $R_{\mathrm{k}^{*} \text {,CD-adapco }}=0.795$ is almost the same magnitude as $R_{\mathrm{k}^{*} \text {,UU-DTU }}$. Similar observations as in test case B are made for the turbulence predictions of CMR. Although the shear layer peaks are on the same level as the experimental values, the levels of turbulence in the wake center and the free stream flow are significantly overpredicted. This observation is confirmed by significantly poorer $\mathrm{MG}_{\mathrm{k}^{*}}$ and $\mathrm{VG}_{\mathrm{k}^{*}}$ than for the other predictions, as shown in the right section of Table 8 .

\section{Discussion and conclusions}

Five different research groups predicted the performance and wake flow between two in-line model wind turbines with a number of different simulation methods. The methods cover different approaches, ranging from commercial software to in-house developed codes. The effects of three different inflow conditions, low-turbulence uniform inflow (test case A), high-turbulence uniform inflow (test case B) and high-turbulence nonuniform shear inflow (test case $\mathrm{C}$ ) are investigated.

The performance of the upstream turbine $\left(C_{\mathrm{P}, \mathrm{T} 1}, C_{\mathrm{T}, \mathrm{T} 1}\right)$ was commonly well predicted by all predictions for all three inlet conditions, with an acceptable scatter of \pm 5 to $\pm 7 \%$ depending on the test case. However, the upstream turbine's performance was well-known from earlier blind tests. The scatter in the performance data of the downstream turbine at design conditions is generally observed to be larger. For $x / D=5.18$ rotor diameter separation distance, the $C_{\mathrm{P}, \mathrm{T} 2}$ predictions varied within $\pm 20 \%$. By decreasing the separation distance to $2.77 D$, the deviations from the measured results were reduced to $\pm 15 \%$, while an increase in separation distance to $9.00 \mathrm{D}$ resulted in an even bigger scatter of $\pm 30 \%$ in all the predictions. The scatter in the downstream turbine thrust coefficient is commonly seen to be smaller than in the power coefficient, while a tendency to underpredict the measured thrust value is observed. Nevertheless, a significant improvement in the predictions downstream of the turbine's performance is observed compared to earlier blind test experiments, in which the scatter was more than $\pm 100 \%$ (Pierella et al., 2014) or $\pm 50 \%$ (Krogstad et al., 2015).

Comparing wake profiles behind the upstream turbine, it can be concluded that both CD-adapco's IDDES computations and UU-DTU's LES simulation consistently deliver very accurate predictions of the experimentally measured mean and turbulent characteristics for all inflow conditions 
and separation distances. CD-adapco and UU-DTU clearly score highest in the statistical correlation coefficients for all the test cases. It seems that CD-adapco's IDDES simulations have a marginally better resolution of flow details, as reflected in very accurate predictions of the shape of the mean velocity and turbulence intensity profiles. This could be due to a better resolution of the small scales in the boundary layers of the rotor, hub and tower geometry, in which the IDDES technique takes advantage of a finer grid resolution in a RANS model. The very precise predictions of the wake shape are also confirmed in a marginally higher score of the correlation coefficients $R_{U^{*}}$ and $R_{\mathrm{k}^{*}}$, which describe correlation of the profile shape well but are insensitive to an offset or multiplication of the data points. Conversely, UU-DTU's simulations predicted the levels of mean velocity deficit slightly better. CD-adapco's mean velocity results have the tendency to predict a marginally too-high velocity deficit, which is reflected in somewhat higher values of the mean geometrical bias $\mathrm{MG}_{U^{*}}$ and geometrical variance $\mathrm{VG}_{U^{*}}$ compared to UU-DTU's generally very precise prediction of the mean velocity levels.

The mean wake profiles are well predicted by the fully resolved $k-\omega$ SST simulations from LUT, whereas the rotorgenerated turbulence in the wake is clearly underpredicted. Simulations by the same group based on an ACL approach are observed not to resolve the flow structures in sufficient detail, which is indicated by somewhat more poorly averaged correlation coefficients $R_{U^{*}}$ and $R_{\mathrm{k}^{*}}$ for the ACL than for the FRR approach.

CMR's wake predictions based on the $k-\varepsilon$ turbulence model mostly manage to approximate the levels of mean velocity deficit reasonably well; however, the details are often lost due to an overprediction of turbulent diffusion. This is also the case for the $k-\omega$ simulations by Vrije, in which acceptable approximations of the mean velocity deficit for high background turbulence inflows are predicted, while the predicted turbulence distributions are observed to be too smooth. The challenges of the more complex nonuniform shear flow were resolved fairly well by most of the simulations since most of them were able to predict a slightly skewed wake.

The discussion in the workshop disclosed that the quality of the wake predictions is dependent not only on the turbulence model, but rather a complex combination of userdependent factors. These could be different methods of meshing, choice of turbulence parameters or force coefficients for rotor modeling, for example. Nevertheless, this blind test also confirms that it is possible to make very accurate performance and wake flow predictions given that the model and input parameters are chosen correctly.

\section{Data availability}

The data presented are available from the authors on request.
Competing interests. The authors declare that they have no conflict of interest.

Acknowledgements. The authors would like to thank the support of NOWITECH for the organization of the workshop.

Edited by: J. Peinke

Reviewed by: three anonymous referees

\section{References}

Adaramola, M. S. and Krogstad, P. Å.: Experimental investigation of wake effects on wind turbine performance, Renew. Energ., 36, 2078-2086, doi:10.1016/j.renene.2011.01.024, 2011.

Ainslie, J. F.: Calculating the flowfield in the wake of wind turbines, J. Wind Eng. Ind. Aerod., 27, 213-224, doi:10.1016/01676105(88)90037-2, 1988.

Aksnes, N. Y.: Performance Characteristics of the NREL S826 Airfoil - An assessment of Re-independency and effect of inflow turbulence, Master's thesis NTNU, 2015.

Barthelmie, R. J., Hansen, K., Frandsen, S. T., Rathmann, O., Schepers, J. G., Schlez, W., Phillips, J., Rados, K., Zervos, A., Politis, E. S., and Chaviaropoulos, P. K.: Modelling and measuring flow and wind turbine wakes in large wind farms offshore, Wind Energy, 12, 431-444, doi:10.1002/we.348, 2009.

Bartl, J. and Sætran, L.: Experimental testing of axial induction based control strategies for wake control and wind farm optimization, J. Phys. Conf. Ser. B, Wind, wakes, turbulence and wind farms, 753, 032035, doi:10.1088/17426596/753/3/032035, 2016.

Bartl, J., Pierella, F., and Sætran, L.: Wake Measurements behind an Array of Two Model Wind Turbines, Energy Procedia, 24, 305-312, doi:10.1016/j.egypro.2012.06.113, 2012.

Benedict, L. and Gould, R.: Towards better uncertainty estimates for turbulence statistics, Exp. Fluids, 22, 129-136, doi:10.1007/s003480050030, 1996.

Bruun, H. H.: Hot-wire anemometry, Principle and signal analysis, Oxford Science Publications, Oxford, UK, ISBN: 9780198563426, 1995.

Chang, J. and Hanna, S.: Air quality model performance evaluation, Meteorol. Atmos. Phys., 87, 167-196, doi:10.1007/s00703-0030070-7, 2004.

Crespo, A., Hernandez, J., Fraga, E., and Andreu, C.: Experimental validation of the UPM computer code to calculate wind turbine wakes and comparison with other models, J. Wind Eng. Ind. Aerod., 27, 77-88, doi:10.1016/0167-6105(88)90025-6, 1988.

Drela, M.: Xfoil 6.99, available at: http://web.mit.edu/drela/Public/ web/xfoil/ (last access: 7 February 2017), 2013.

Eriksen, P. E.: Rotor wake turbulence - An experimental study of a wind turbine wake, Doctoral thesis at NTNU, 34, ISBN 978-82326-1408-0, 2016.

Frandsen, S., Barthelmie, R., Pryor, S., Rathmann, O., Larsen, S., Højstrup, J., and Thøgersen, M.: Analytical modelling of wind speed deficit in large offshore wind farms, Wind Energy, 9, 3953, doi:10.1002/we.189, 2006.

Hallanger, A. and Sand, I. Ø.: CFD Wake modelling with a BEM wind turbine sub-model, Modeling, Identification and Control, 34, 19-33, ISSN 1890-1328, 2013. 
Hsu, S. A., Meindl, E. A., and Gilhousen, D. B.: Determining the power-law wind-profile exponent under near-neutral stability conditions at sea, J. Appl. Meteorol., 33, 757-765, doi:10.1175/1520-0450(1994)033<0757:DTPLWP>2.0.CO;2, 1994.

Jensen, N. O.: A note on wind generator interaction, Technical Report, Ris $\varnothing$, RIS $\varnothing-\mathrm{M}-2411$, available at: www.orbit.dtu.dk/fedora/ objects/orbit:88807 (last access: 7 February 2017), 1983.

Kocer, G., Mansour, M., Chokani. N., Abhari, R. S., and Müller, M.: Full-Scale Wind Turbine Near-Wake Measurements Using an Instrumented Uninhabited Aerial Vehicle, J. Sol. Energy Eng., 133, 041011, doi:10.1115/1.4004707, 2011.

Kumer, V. M., Reuder, J., Svardal, B., Sætre, C., and Eecen, P.: Characterisation of Single Wind Turbine Wakes with Static and Scanning WINTWEX-W LiDAR Data, Energy Procedia, 80, 245-254, doi:10.1016/j.egypro.2015.11.428, 2015.

Krogstad, P. A. and Eriksen, P. E.: "Blind test" calculations of the performance and wake development for a model wind turbine, Renew. Energ., 50, 325-333, doi:10.1016/j.renene.2012.06.044, 2013.

Krogstad, P. Å., Sætran, L., and Adaramola, M. S.: Blind Test 3 calculations of the performance and wake development behind two in-line and offset model wind turbines, J. Fluid. Struct., 52, 65-80, doi:10.1016/j.jfluidstructs.2014.10.002, 2015.

Larsen, G. C., Madsen, H. A., Larsen, T. J., and Troldborg, N.: Wake modeling and simulation, Technical Report, Ris $\emptyset-\mathrm{R}-1653(\mathrm{EN})$, available at: www.orbit.dtu.dk/fedora/objects/orbit:80992 (last access: 7 February 2017), 2008.

Ning, S. A.: A simple solution method for the blade element momentum equations with guaranteed convergence, Wind Energy, 17, 1327-1345, doi:10.1002/we.1636, 2014.

Maal, G. T.: Parameter study of electric power production in wind farms - experiments using two model scale wind turbines, Master's thesis NTNU, available at: https://brage.bibsys.no/xmlui/bitstream/handle/11250/2350000/ 12371_FULLTEXT.pdf?sequence=1 (last access: 7 February 2017), 2014.

Medici, D. and Alfredsson, P. H.: Measurements on a Wind Turbine Wake: 3-D Effects and Bluff Body Vortex Shedding, Wind Energy, 9, 219-236, doi:10.1002/we.156, 2006.

Mendonça, F., Baris, O., and Capon, G.: Simulation of Radial Compressor Aeroacoustics Using CFD, ASME Turbo Expo 2012, Turbine Technical Conference and Exposition, Copenhagen, Denmark, 8, 1823-1832, Paper No. GT2012-70028, doi:10.1115/GT2012-70028, 2012.

Ostovan, Y., Amiri, H., and Uzol, O.: Aerodynamic Characterization of NREL S826 Airfoil at Low Reynolds Numbers, RUZGEM Conference on Wind Energy Science and Technology, METU Ankara Campus, 3-4 October 2013, Ankara, Turkey, 2013.

Pierella, F., Krogstad, P. Å., and Sætran, L.: Blind Test 2 calculations for two in-line model wind turbines where the downstream turbine operates at various rotational speeds, Renew. Energ., 70, 62-77, doi:10.1016/j.renene.2014.03.034, 2014.

Prytz, E., Huuse, Ø., Müller, B., Bartl, J., and Sætran, L.: Numerical Simulation of Flow around the NREL S826 Airfoil at Moderate Reynolds numbers Using Delayed Detached Eddy Simulation (DDES), Proceedings ICNAAM 2016, ICNAAM_2016_124, accepted, 2017.
Sætran, L. and Bartl, J.: Invitation to the 2015 "Blind test 4" Workshop, Technical document NTNU, www.sintef.no/globalassets/ project/nowitech/events/bt4_invitation2015.pdf (last access: 7 February 2017), 2015.

Sagmo, K. F., Bartl, J., and Sætran, L.: Numerical simulations of the NREL S826 airfoil, J. Phys. Conf. Ser., 753, 082036, doi:10.1088/1742-6596/753/8/082036, 2016.

Sanderse, B., van der Pijl, S. P., and Koren, B.: Review of computational fluid dynamics for wind turbine wake aerodynamics, Wind Energy, 14, 799-819, doi:10.1002/we.458, 2011.

Sarmast, S. and Mikkelsen, R.: The experimental results of the NREL S826 airfoil at low Reynolds numbers, Technical Report, available at: http://www.diva-portal.org/smash/record.jsf? pid=diva2\%3A615785\&dswid=-528 (last access: 7 February 2017), 2013.

Sarmast, S., Dadfar, R., Mikkelsen, R. F., Schlatter, P., Ivanell, S., Sørensen, J. N., and Henningson, D. S.: Mutual inductance instability of the tip vortices behind a wind turbine, J. Fluid Mech., 755, 705-731, doi:10.1017/jfm.2014.326, 2014.

Schepers, J. G., Boorsma, K., and Snel, H.: IEA Task 29 MexNext: analysis of wind tunnel measurements from the EU project Mexico, Proceedings of The Science of Making Torque from Wind, 169-178, 2010.

Schepers, J. G., Boorsma, K., and Munduate, X.: Final Results from Mexnext-I: Analysis of detailed aerodynamic measurements on a $4.5 \mathrm{~m}$ diameter rotor placed in the large German Dutch Wind Tunnel DNW, J. Phys. Conf. Ser., 555, 012089, doi:10.1088/1742-6596/555/1/012089, 2014.

Schümann, H., Pierella, F., and Sætran, L.: Experimental Investigation of Wind Turbine Wakes in the Wind Tunnel, Energy Procedia, 35, 285-296, doi:10.1016/j.egypro.2013.07.181, 2013.

Somers, D. M.: The S825 and S826 Airfoils, Technical Report NREL/SR-500-36344, available at: https://wind.nrel.gov/ airfoils/Documents/S825,S826_Design.pdf (last access: 7 February 2017), 2005.

Sørensen, J. N. and Shen, W. Z.: Numerical modeling of wind turbine wakes, J. Fluid Eng., 124, 393-399, doi:10.1115/1.1471361, 2002.

Talmon, A. M.: The wake of a horizontal-axis wind turbine model, measurements in uniform inflow and in a simulated atmospheric boundary layer, TNO Data report, available at: http://publications.tno.nl/publication/100176/YosHZP/ 85-010121.pdf (last access: 7 February 2017), 1985.

Trujillo, J. J., Seifert, J. K., Würth, I., Schlipf, D., and Kühn, M.: Full-field assessment of wind turbine near-wake deviation in relation to yaw misalignment, Wind Energ. Sci., 1, 41-53, doi:10.5194/wes-1-41-2016, 2016.

Van Leer, B: Towards the ultimate conservative differencing scheme II: Monotonicity and conservation combined in a second-order scheme, J. Comput. Phys., 14, 361-370, 1974.

Wagner, R., Cañadillas, B., Clifton, A., Feeney, S., Nygaard, N., Poodt, M., St. Martin, C., Tüxen, E., and Wagenaar, J. W.: Rotor equivalent wind speed for power curve measurement - comparative exercise for IEA Wind Annex 32, J. Phys. Conf. Ser., 524, 012108, doi:10.1088/1742-6596/524/1/012108, 2014.

Wheeler, A. J. and Ganji, A. R.: Introduction to engineering experimentation, Third edition, Upper Saddle River, NJ, USA, Pearson/Prentice Hall, XI, ISBN-13:987-0-13-511314-1, 2004. 